\title{
The correlation between photometric variability and radial velocity jitter
}

\section{Based on TESS and HARPS observations *}

\author{
S. Hojjatpanah ${ }^{1,2}$, M. Oshagh ${ }^{3,4,1}$, P. Figueira ${ }^{5,1}$, N. C. Santos ${ }^{1,2}$, E. M. Amazo-Gómez ${ }^{6,4}$, S. G. Sousa ${ }^{1}$, \\ V. Adibekyan ${ }^{1,2}$, B. Akinsanmi ${ }^{1,2,7}$, O. Demangeon ${ }^{1}$, J. Faria ${ }^{1}$, J. Gomes da Silva ${ }^{1}$, and N. Meunier ${ }^{8}$ \\ ${ }^{1}$ Instituto de Astrofísica e Ciências do Espaço, Universidade do Porto, CAUP, Rua das Estrelas, 4150-762 Porto, Portugal \\ e-mail: saeed.hojjatpanah@astro.up.pt \\ ${ }^{2}$ Departamento de Fisica e Astronomia, Faculdade de Ciências, Universidade do Porto, Rua Campo Alegre, $4169-007$ Porto, \\ Portugal \\ ${ }^{3}$ Instituto de Astrofísica de Canarias (IAC), 38200 La Laguna, Tenerife, Spain \\ ${ }^{4}$ Institut für Astrophysik, Georg-August-Universität, Friedrich-Hund-Platz 1, 37077 Göttingen, Germany \\ ${ }^{5}$ European Southern Observatory, Alonso de Cordova 3107, Vitacura, Santiago, Chile \\ ${ }^{6}$ Max-Planck-Institut fur Sonnensystemforschung, Göttingen, Germany \\ ${ }^{7}$ National Space Research and Development Agency. Airport Road, Abuja, Nigeria \\ ${ }^{8}$ Univ. Grenoble Alpes, CNRS, IPAG, 38000 Grenoble, France
}

Received 27 March 2020 / Accepted 15 May 2020

\begin{abstract}
Context. Characterizing the relation between stellar photometric variability and radial velocity (RV) jitter can help us to better understand the physics behind these phenomena. The current and upcoming high precision photometric surveys such as TESS, CHEOPS, and PLATO will provide the community with thousands of new exoplanet candidates. As a consequence, the presence of such a correlation is crucial in selecting the targets with the lowest RV jitter for efficient RV follow-up of exoplanetary candidates. Studies of this type are also crucial to design optimized observational strategies to mitigate RV jitter when searching for Earth-mass exoplanets.

Aims. Our goal is to assess the correlation between high-precision photometric variability measurements and high-precision RV jitter over different time scales.

Methods. We analyze $171 \mathrm{G}, \mathrm{K}$, and M stars with available TESS high precision photometric time-series and HARPS precise RVs. We derived the stellar parameters for the stars in our sample and measured the RV jitter and photometric variability. We also estimated chromospheric Ca II H \& K activity indicator $\log \left(R_{\mathrm{HK}}^{\prime}\right), v \sin i$, and the stellar rotational period. Finally, we evaluate how different stellar parameters and an RV sampling subset can have an impact on the potential correlations.

Results. We find a varying correlation between the photometric variability and RV jitter as function of time intervals between the TESS photometric observation and HARPS RV. As the time intervals of the observations considered for the analysis increases, the correlation value and significance becomes smaller and weaker, to the point that it becomes negligible. We also find that for stars with a photometric variability above $6.5 \mathrm{ppt}$ the correlation is significantly stronger. We show that such a result can be due to the transition between the spot-dominated and the faculae-dominated regime. We quantified the correlations and updated the relationship between chromospheric Ca II H \& $\mathrm{K}$ activity indicator $\log \left(R_{\mathrm{HK}}^{\prime}\right)$ and $\mathrm{RV}$ jitter.
\end{abstract}

Key words. techniques: spectroscopic - planets and satellites: detection - techniques: radial velocities - techniques: photometric stars: activity

\section{Introduction}

Detecting and accurately characterizing low-mass exoplanets is a challenge for observational astronomy. Besides instrumental limitations, one of the main remaining obstacles is the intrinsic stellar variability, often called "jitter" in the exoplanet's field. The two main detection methods, radial velocity (RV) and transit photometry, struggle with the impact of stellar activity induced signal (e.g., Oshagh 2018), which is generated by active regions, such as spots and plages, associated to strong magnetic fields. The presence of active regions on a rotating star not only

\footnotetext{
${ }^{\star}$ Based on observations collected at the La Silla Observatory, ESO(Chile), with the HARPS spectrograph at the 3.6-m telescope. See the acknowledgements for the list of specific programs.
}

generates RV jitter but also induces photometric modulations in transit observations (e.g., Czesla et al. 2009; Oshagh 2018).

Stellar activity can both mimic RV planetary signals (similar to planetary signals) and hide a real planetary signal by adding significant stellar activity induced signal. (e.g., Saar et al. 1998; Dumusque et al. 2011a,b; Queloz et al. 2001; Santos et al. 2002, 2010, 2014; Boisse et al. 2011a; Díaz et al. 2016). Moreover, it can degrade the precision of the planetary parameters derived through RV (e.g., Cloutier et al. 2018; Fischer et al. 2016) or transit measurements (Czesla et al. 2009; Oshagh et al. 2013a,b, 2015; Barros et al. 2014).

Huélamo et al. (2008) and Figueira et al. (2010) demonstrated that the stellar spots can even reproduce the RV variations as high as those produced by giant planets on short orbits. Additionally, Robertson et al. (2014) showed that the activity can 
masquerade as a planet located inside the habitable zone of M dwarfs.

It has further been shown that the stellar photometric variability is correlated with the RV jitter (e.g., Aigrain et al. 2012; Bastien et al. 2014; Oshagh et al. 2017). Defining such relations as precisely as possible is crucial in optimizing the telescope time invested in the RV follow-up of upcoming planetary candidates from ongoing and upcoming missions such as TESS (Ricker et al. 2014a) and PLATO (Rauer et al. 2014) with high-resolution spectrographs, such as HARPS (Mayor et al. 2003) and ESPRESSO (Pepe et al. 2014). Several studies have attempted to assess this potential correlation. Bastien et al. (2014) compared the variability in Kepler light-curves and the root-mean-square (RMS) of RV time series of twelve $\mathrm{G}$ and $\mathrm{K}$ stars. They did not find any strong correlation between the RV-RMS and the amplitude of photometric variability. Instead, they found a correlation between the number of significant peaks in the Lomb-Scargle periodograms of the light-curves and the RMS of stellar RV.

Later, Cegla et al. (2014) studied a sample of 900 stars using GALEX (Martin et al. 2005) and Kepler surveys (Borucki et al. 2010). They estimated the chromospheric activity index of each target using the UV flux measured by GALEX (Findeisen et al. 2011) and used it to calculate the RV jitter by using empirical relations reported in several studies (Santos et al. 2000; Wright 2005; Saar et al. 2003). For magnetically quiet stars, they found a strong correlation between photometric variability and the estimated RV jitter. They also evaluated the correlation between RV jitter and $F_{8}{ }^{1}$ (Bastien et al. 2013) and additionally with the number of zero crossings (X0) in photometric observation. They found that the correlation between RV jitter and $F_{8}$ is sensitive to the effective temperature of the stars.

Oshagh et al. (2017) performed an intensive study on a sample of nine stars with different levels of activity. They obtained simultaneous K2 photometric light-curves and HARPS RV measurements. From these observations the photometric variability, $F_{8}$, RV jitter, spectroscopic activity indicators BIS, FWHM, and $\log \left(R_{\mathrm{HK}}^{\prime}\right)$ were derived. The authors found that a strong correlation exists between RV jitter and photometric variation for highly active stars $\left(\log \left(R_{\mathrm{HK}}^{\prime}\right) \geq-4.66\right)$. Moreover they found that for highly active stars strong correlations exist between RV jitter and variability of activity indicators, such as BIS, FWHM, and $\log \left(R_{\mathrm{HK}}^{\prime}\right)$. The authors pointed out that all of these correlations become weaker for stars with low-amplitude photometric variations and for slowly-rotating stars. The same study also showed that even though $F_{8}$ has a strong correlation with the RV jitter, it does not display a strong correlation with rotationally-induced photometric variability. They also confirmed the evidence of the two spot-dominated and plage-dominated regimes presented in several other studies (Lockwood et al. 1997; Radick et al. 1998; Shapiro et al. 2016).

Recently, Tayar et al. (2019) investigated the relationship between stellar properties (such as $\log g$, temperatures, and metallicities) and convective granulation using $F_{8}$. They used a large sample of dwarfs, subgiants and red giants (2465 stars in total) observed by Kepler and APOGEE surveys. With their study, they updated the relationship between convective granulation and stellar properties. Using the empirical Flicker-jitter relations presented by Bastien et al. (2014); Oshagh et al. (2017), they predicted the granulation-driven RV jitter amplitudes for a sample of stars observed by TESS.

$1 F_{8}$ is the RMS of photometric light-curve on timescale shorter than $8 \mathrm{hr}$, and has been shown to also correlate strongly with the stellar $\log g$.
The main objective of the current study is to extend these studies to explore, in unprecedented detail, the existence of correlations between RV jitter and photometric variability measurements. This study is performed for $171 \mathrm{G}, \mathrm{K}$ and $M$ dwarfs observed with the high-resolution and stable spectrograph HARPS and with the TESS mission, which both provide some of the best RV and photometric precision available.

This paper is organized as following: we explain the target selection and data reduction in Sect. 2. The derivation of stellar parameters is described in Sect. 3. In Sect. 4, we analyze the correlation between RV jitter and photometric peak-to-peak variation. The application of our obtained result on TESS planet candidate host stars is presented in Sect. 6 by identifying the potential targets which will have the lowest RV jitter based on their light curve photometric variability. Finally, conclusions are drawn in Sect. 7.

\section{Target selection and data reduction}

\subsection{Target selection}

We selected our targets by applying the following criteria: (a) Cross matching the stars in the HARPS archive with those observed by TESS during its first year (covering 13 observation sectors in the Southern Ecliptic Hemisphere) ${ }^{2}$. All targets have their RV and photometric observations publicly available in the ESO archive and Mikulski Archive for Space Telescopes $(\mathrm{MAST})^{3}$, respectively. (b) Selecting only the stars with RV measurements taken after the fiber change of HARPS on June 2015 (Lo Curto et al. 2015) to avoid offset in RV measurements and to make the RV measurements as homogeneous as possible ${ }^{4}$. (c) Removing the targets with known exoplanets to eliminate RV variations due to the known companions ${ }^{5}$. (d) Selecting stars with measurements on at least 5 nights over a minimum period of one week ${ }^{6}$. This criterion aims at producing a minimum sampling of the rotation modulation of $\mathrm{G}, \mathrm{K}$ and $\mathrm{M}$ dwarfs (Nielsen et al. 2013; Reinhold \& Hekker 2020).

A total of 171 stars satisfy all of the above criteria. They are all listed in Table. C.1. In Fig. 1, we illustrated the TESS observation windows (sectors) and RV measurement epochs of each target.

\subsection{Light curve: TESS}

We obtained the light-curves of all 171 stars from MAST. MAST contains simple aperture photometry (SAP_flux; Morris et al. 2017) as well as presearch data conditioning (PDCSAP_flux). Most of the targets (96\%) were observed in only one TESS sector. For stars with light-curves in two or more consecutive sectors, we merged all available light-curves. We used qualityflag $^{7}$ as suggested by the TESS Data Product review, also recently used and tested in Vida et al. (2019). We used SAP_flux which optimizes the aperture for the best signal-to-noise for the each target (Bryson et al. 2017) and also the calibrated pixels in

\footnotetext{
2 https://tess.mit.edu/observations/

3 https://archive.stsci.edu

4 Including the data before the fiber change would add few points per star and one free parameter, the offset between the two, which brings only a little gain.

5 https://exoplanetarchive.ipac.caltech.edu exoplanet. eu

6 We have applied this criteria on the reduced final RV measurements which we explained in Sect. 2.3.

101010111111: https://outerspace.stsci.edu/display/ TESS/2.0+-+Data+Product+Overview
} 


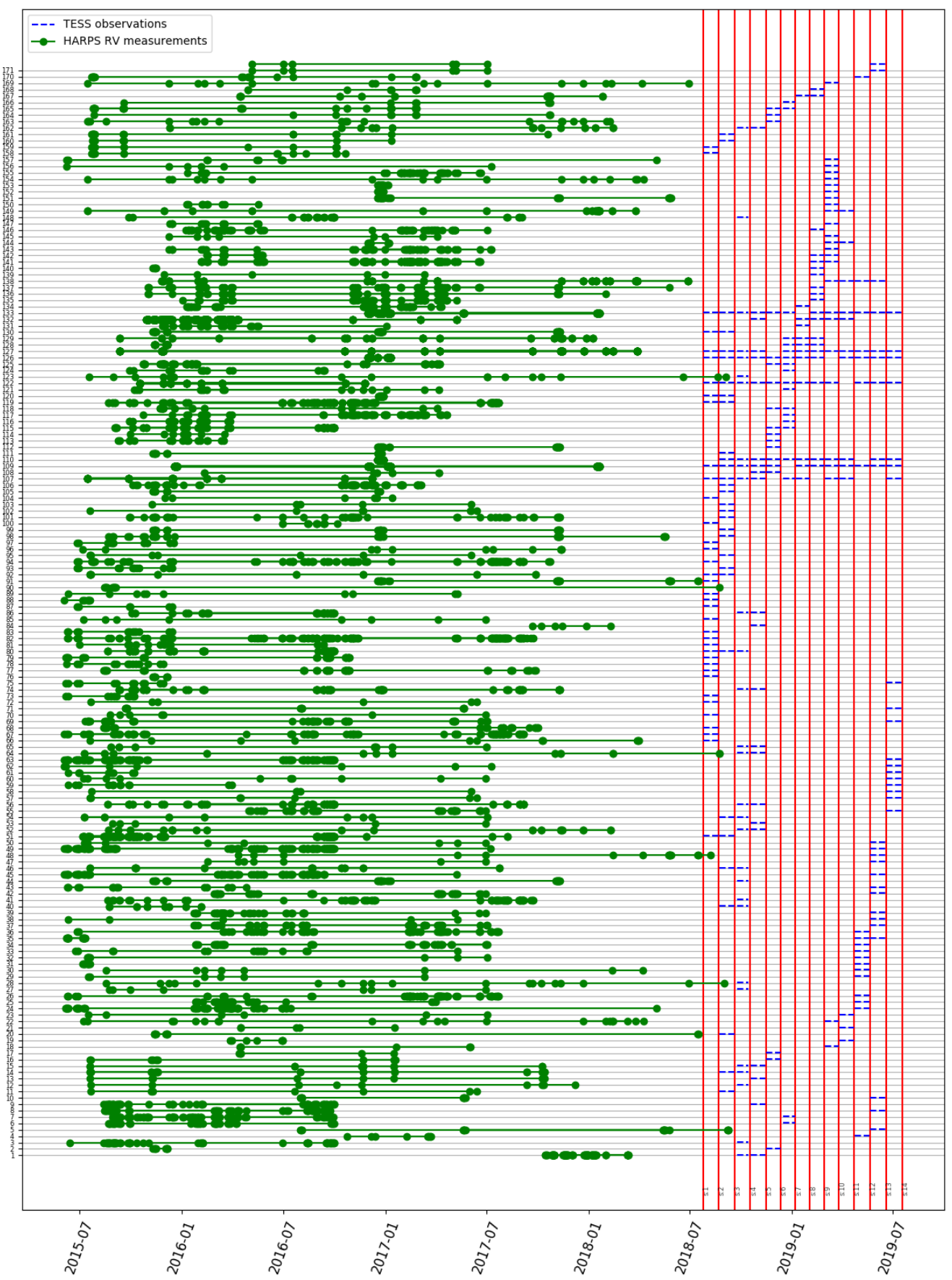

Fig. 1. Total RV measurements and TESS observation windows. The green points show the RV measurements dates. Blue dash and red vertical lines show the TESS observations and sectors, respectively. The name of each star is presented in Table. C.1 using index number in $y$-axis.

order to perform a summation of the flux. The SAP light curves provided by the TESS pipeline are also background corrected. We removed outlier flux points using a sigmaclipping of three standard deviations and smoothed the fluxes using SavitzkyGolay filter within 15 data point windows $(\sim 30 \mathrm{~min})$ to reduce the effect of the short-time scale photometric variability. Since we are interested in variability at stellar rotation timescales, this smoothing does not affect our results. We then normalized the flux by the median flux values. We derived the ratio between the peak-to-peak of light curve variability of SAP_flux and the peak-to-peak of light curve variability of PDCSAP_flux. If this ratio was larger than 3.0, we checked the light curves visually to ensure if there was any evidence for systematic errors in
SAP_flux. For more than $90 \%$ of the stars, we used SAP_flux, and for the rest we assumed that the light-curves are dominated by systematic errors therefore we used PDCSAP_FLUX. In Fig. 2, you can see a sample of reduced light curve in one sector for one star.

\subsection{HARPS RVs}

For the 171 stars selected in Sect. 2 we collected the available HARPS data through ESO archive ${ }^{8}$. The data were reduced using the official HARPS pipeline (DRS), that delivers the RV

\footnotetext{
8 https://www.eso.org/sci/observing/phase3/data_ streams.html
} 


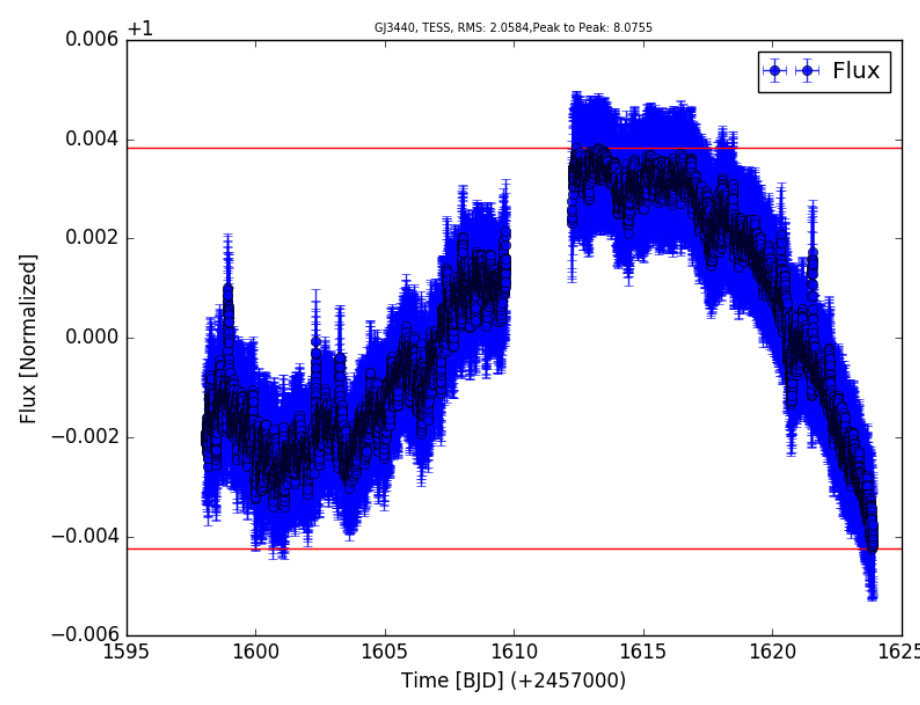

Fig. 2. Example of light curve of GJ3440 during one TESS sector (blue points) and the red lines presenting the peak-to-peak of light curve.

and full width at half maximum (FWHM) measured on each Cross-Correlation Function (CCF). We determined the significant outliers of the RV time-series by applying a sigmaclipping of three standard deviations, and removed the few measurements that show an RV error larger than $9 \mathrm{~ms}^{-1}$ as an admittedly arbitrary value. This threshold was set for being significantly larger than the median RV error $\left(\sim 0.80 \mathrm{~ms}^{-1}\right)$ for the whole sample. After the nightly binning of the data, in total, over 3971 spectra were collected and $81 \mathrm{RV}$ measurements $(\sim 2 \%)$ were removed based on the previous outlier and photon noise criteria. For each star, we subtracted the mean value of the RV from the RV measurements. Moreover, we corrected for linear long term RV trends using a linear fit for 27 stars of our sample ${ }^{9}$. This linear trend removal was done to ensure eliminating the signal from any possible unknown long period companions around our targets. It is worth mentioning that unknown planets on short orbits could also affect our RV-RMS measurements, however, if undiscovered and passing the previous vetting criteria, it means that they must be low mass planets and induce low RV semi-amplitude, and thus they do not affect our study significantly. In Fig. 3, we present an example of final RV time-series of one star as well as its associated RV-RMS measurement. Minimum, maximum, and the average of the time spans of the RV observations are $\sim 7$ days, $\sim 3 \mathrm{yr}$, and $1.5 \mathrm{yr}$, respectively. The histogram of the RV time spans is presented in Fig. B.4.

\subsection{Subsamples}

The HARPS measurement are not contemporaneous with TESS observations. Since stellar activity patterns are expected to evolve with time, we divided our whole sample into 4 subsamples as a function of time lag between TESS and HARPS. For the first subsample we considered only the stars with RVs measured up to one year prior to the TESS observations, hereafter named as "year 1". The second subsample contains the stars that have RV measurements in a time interval from one year to two

9 GJ 3440, HD 021175, HD 107094, HD 1388, HD 16548, HD 185283 , HD 19230, HD 19641, HD 200133, HD 202917, HD 205536, HD 207129, HD 211415, HD 213042, HD 218860, HD 221638, HD 222335, HD 28471, HD 3074A, HD 40397, HD 42936, HD 6107, HD 76849, HD 88218, HD 89839, HD 94771, HD 96700.

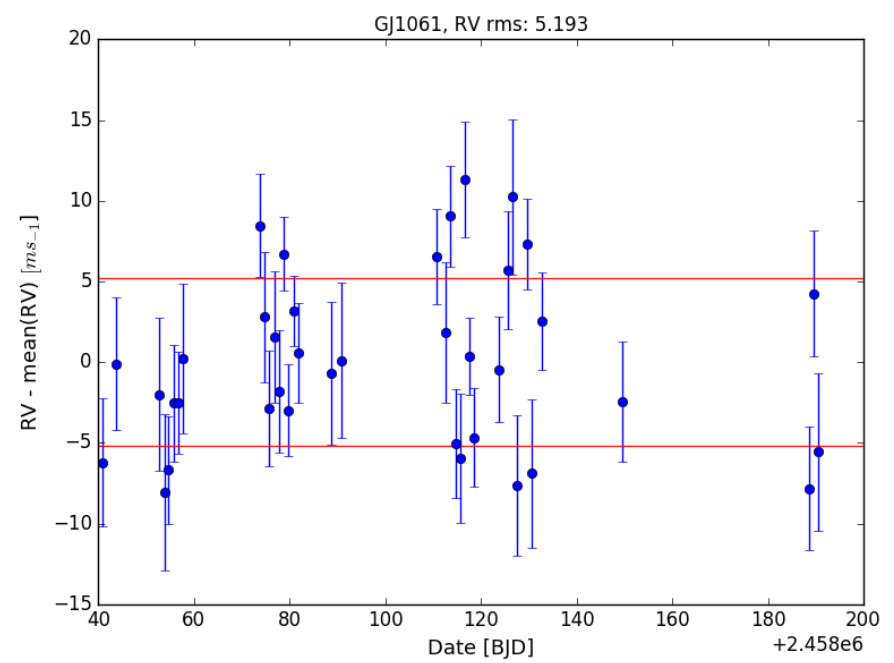

Fig. 3. Example of RV measurements for GJ 1061 (blue dots), and the red lines presenting the \pm RV-RMS values.

years before TESS observations, hereafter named as "year 2"; the same criteria were used to create the third and the fourth subsamples hereafter named as "year 3" and "year 4", respectively. Thus, it is important to emphasize that we divided all the RV measurements into four subsamples, and some stars are present in more than one subsample. According to our criteria for dividing the whole RV measurements into the four subsamples, few stars were not categorized in any of these four subsamples, and are only considered in the whole sample. Despite the ability for a star to appear in multiple subsamples, the RV-RMS for a star in a given subsample only uses the RVs taken during that subsample's time-frame. The samples in which targets are present is listed in Table. C.1; stars which are used only in whole sample are labeled as "w".

\section{Stellar parameters}

Accurate stellar parameters are necessary for the characterization of exoplanets and their hosts (Mayor et al. 2004; Fischer \& Valenti 2005; Reffert et al. 2015; Adibekyan et al. 2018). Stellar parameters rule the physics behind the generation of stellar activity regions, and are thus of interest in this analysis. Therefore, we derived the main spectroscopic stellar parameters: effective temperature $\left(T_{\text {eff }}\right)$, metallicity $([\mathrm{Fe} / \mathrm{H}])$, surface gravity $(\log g)$ and stellar rotation rate for the stars in our sample. The stellar parameters for $\mathrm{G}$ and $\mathrm{K}$ dwarfs are determined with the procedure described in Sousa (2014). For the M dwarfs in the sample, we used a modified version of the method and software developed by Neves et al. $(2014)^{10}$.

Figure 4 presents the Hertzsprung-Russell diagram (HR), for the stars in our sample on which we over-plotted the samples that were used in the two similar previous studies (Oshagh et al. 2017; Bastien et al. 2014). Our sample size is one order of magnitude larger than the previous studies, and covers a wider range of effective temperatures for $\mathrm{G}$ and $\mathrm{K}$ dwarfs; however, the number of M-dwarfs in our sample is relatively small.

\subsection{Chromospheric activity indicator $\log \left(R_{H K}^{\prime}\right)$ and $v \sin i$}

We derived values for the S-index of each star to evaluate the chromospheric magnetic activity level and its evolution. We used

10 http://WwW . astro.up.pt/resources/mcal/Site/MCAL .html 


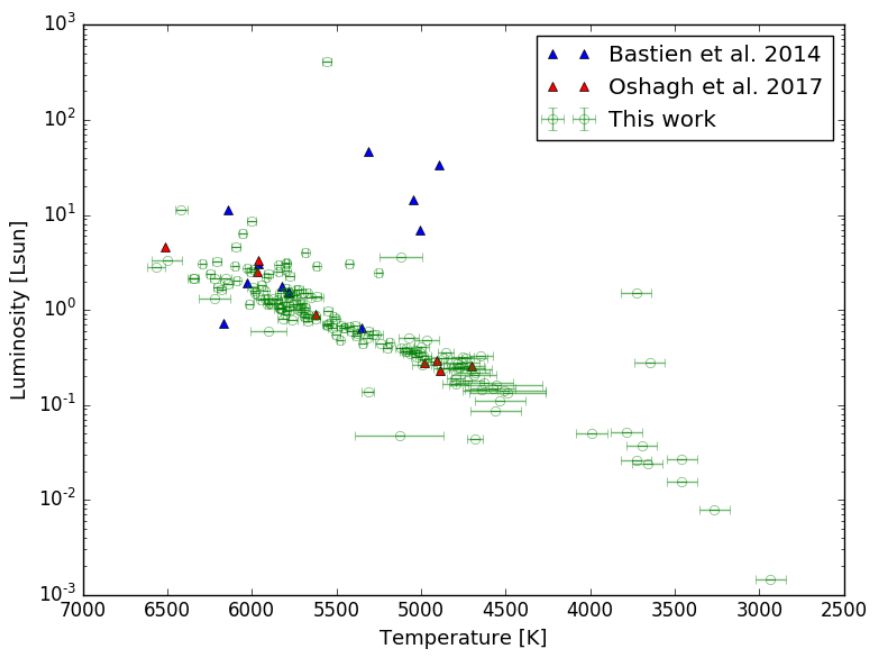

Fig. 4. HR diagram for the sample of stars used in this work.

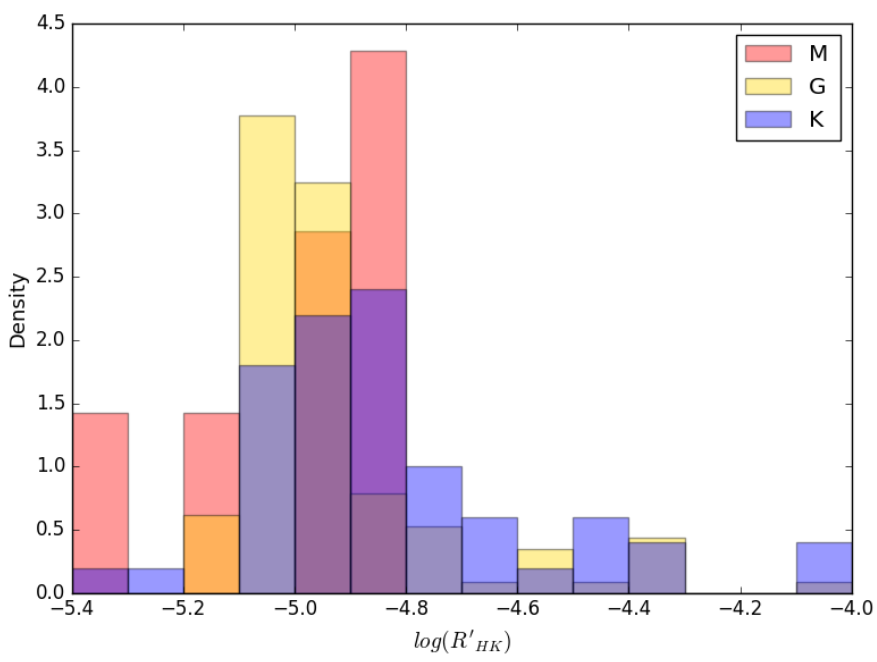

Fig. 5. Histogram of mean $\log \left(R_{\mathrm{HK}}^{\prime}\right)$ for the sample of stars used in this work. Red, gold and blue correspond to the spectral type of the star, M, $\mathrm{G}$ and $\mathrm{K}$ respectively.

the open source package $A C T I N^{11}$ (Gomes da Silva et al. 2018) to calculate the $S_{\text {CaII }}$ index (Vaughan et al. 1978; Duncan et al. 1991) homogeneously for all individual spectra following the instructions in (Gomes da Silva et al. 2011; Hojjatpanah et al. 2019 , and references therein). We then calibrated $S_{\text {CaII }}$ to the Mt. Wilson scale using open source package pyrhk $k^{12}$ and calculated the $R_{\mathrm{HK}}^{\prime}$ chromospheric emission ratio as defined in Noyes et al. (1984).

For each star we created a $\log \left(R_{\mathrm{HK}}^{\prime}\right)$ time-series and calculated the mean and peak-to-peak of variation of $\log \left(R_{\mathrm{HK}}^{\prime}\right)$ to evaluate the variation of chromospheric activity during the observation span. In Fig. 5 we present the distribution of mean $\log \left(R_{\mathrm{HK}}^{\prime}\right)$ as a function of spectral types. This figure shows that the majority of our stars are magnetically relativity similar to the solar average value of $\log \left(R_{\mathrm{HK}}^{\prime}\right) \sim-4.90$ (Mamajek \& Hillenbrand 2008), which is a consequence of a bias in the target selection of stars for precise RV searches with HARPS.

\footnotetext{
${ }^{11}$ https://pypi.org/project/actin/, https://github.com/ gomesdasilva/ACTIN

12 https://github.com/gomesdasilva/pyrhk
}

It has been demonstrated that the RV jitter depends strongly on the projected rotational velocity $(v \sin i)$ (e.g., Saar \& Donahue 1997; Desort et al. 2007; Boisse et al. 2011b; Korhonen et al. 2015). Thus, we expect that $v \sin i$ should be one of the influential parameters in this investigation. Therefore, we estimated $v \sin i$ of all our stars based on the measured FWHM of the CCF. We used the procedure described in Santos et al. (2002); Maldonado et al. (2017); Hojjatpanah et al. (2019) and references therein.

\subsection{Stellar rotation period}

Photometric contrast differences associated to magnetic features (e.g., dark spots and bright faculae) generate traceable signatures of stellar rotation periods on light curves.

We analyze the presence of a periodic modulation signal from stellar rotation on the TESS photometric time-series using the gradient of the power spectra (GPS; see Shapiro et al. 2020; Amazo-Gómez et al. 2020). We successfully recover the rotation period for 71 out of 171 stars of the sample. We report the estimated rotation period from the GPS method in Table. C.1. The rotation period from GPS is determined from the enhanced profile of the high-frequency tail of the power spectrum. In particular, we identify the point where the gradient of the power spectrum GPS in $\log -\log$ scale reaches its maximum value. Such a point corresponds to the high frequency inflection point (HFIP), that is, where the concavity of the power spectrum plotted in the log-log scale changes sign. The position of inflection point is related to the rotation period of star by the calibration factor $\alpha_{\text {Sun }}$, for Sun-like stars.

For the calculations presented in this project we adopt a solar-like calibration factor $\alpha_{\text {Sun }} \pm 2 \sigma=0.158 \pm 0.014$, and 2 sigma uncertainty (for more details see Shapiro et al. 2020; Amazo-Gómez et al. 2020).

We also estimated the faculae to spot ratio for 29 of the 71 stars. Following Shapiro et al. (2020); Amazo-Gómez et al. (2020), the light curve is faculae dominated when the ratio between HFIP and the independent rotation period ranges between [0.11 and 0.16], and spot is dominated when the value falls between [0.16 and 1.24].

\section{Correlation between RV jitter and photometric peak-to-peak}

\subsection{Correlation as a function of time}

In Fig. 6 we present the RV-RMS versus peak-to-peak of the photometric time series for the four different subsamples. To evaluate the possible influence of stellar rotation velocity and its magnetic activity level variation, we code these variables into marker color and size.

In order to evaluate the strength of the correlation between RV-RMS and peak-to-peak of light curve, we derived two correlation coefficients. We used the Pearson's correlation coefficient $\left(\rho_{\text {pe }}\right)$ that evaluates the presence of a linear relationship between data pairs, as well as Spearman's correlation coefficient $\left(\rho_{\mathrm{sp}}\right)$ that, working on ranked data, assesses the presence of a monotonic relationship. Both correlation coefficients were derived using the code described in Figueira et al. (2016) which uses the Bayesian framework to evaluate the significance of the presence of a correlation.

In each panel of Fig. 6 we also report the value of the $\rho_{\text {pe }}$ correlation coefficient and its posterior distribution. For data obtained within year 1 time span, we obtained the correlation 


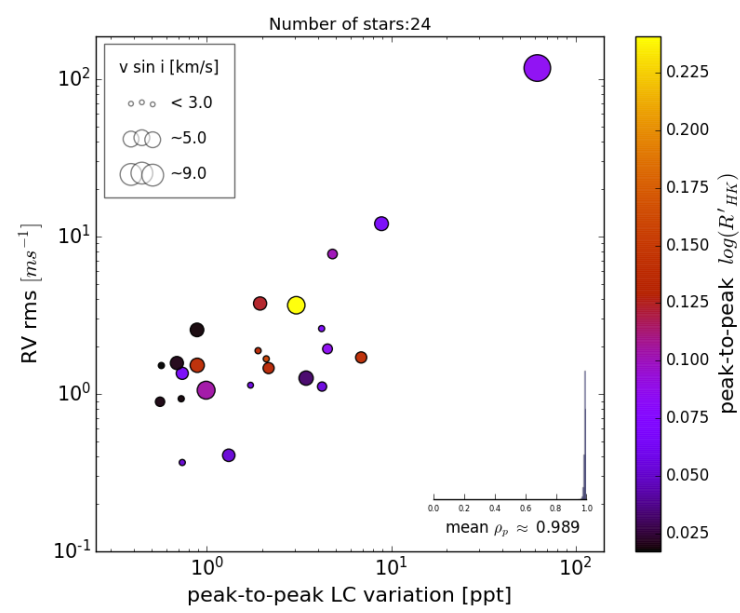

(a) year 1

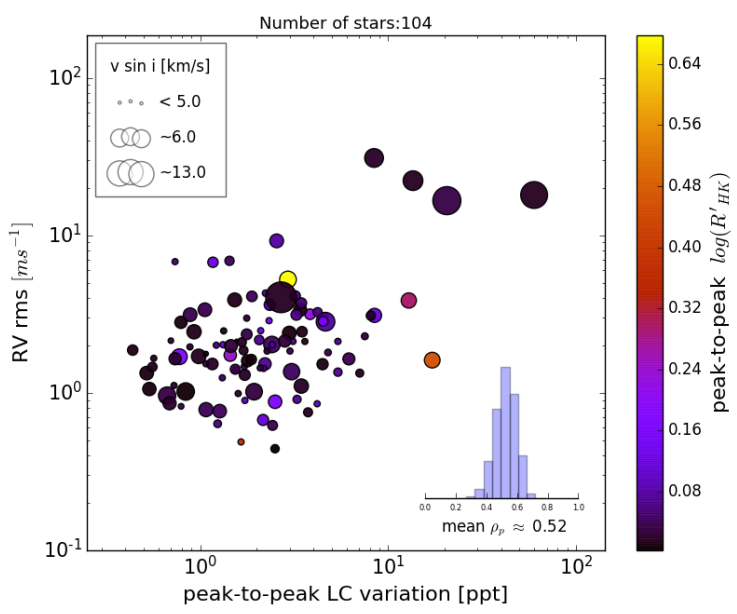

(c) year 3

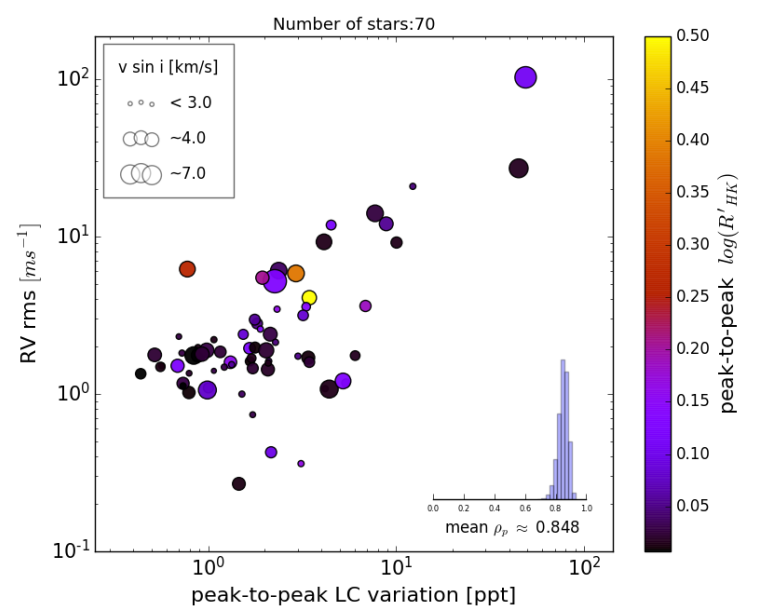

(b) year 2

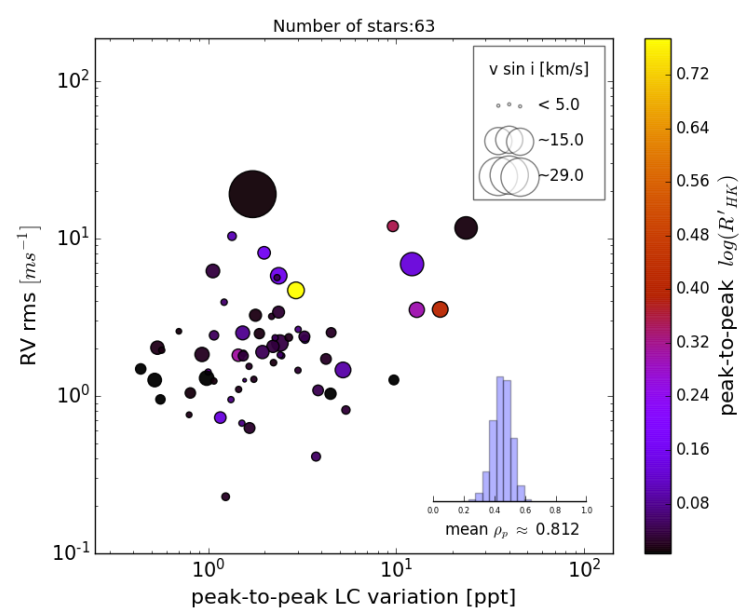

(d) year 4

Fig. 6. RV-RMS versus photometric variability (peak-to-peak of light curve) for the year $1(a)$, year $2(b)$, year $3(c)$ and year $4(d)$ RV measurements sampling. Color bars present the peak-to-peak variation of $\log \left(R_{\mathrm{HK}}^{\prime}\right)$ during the whole HARPS observations and the size-scale presents the estimation of the average $v \sin i$ derived from the CCF FWHM described in Sect. 3.1. The calculated value of correlation coefficient $\rho_{\mathrm{sp}}$ and its posterior distribution is presented in each panel.

Table 1. Result for Pearson's correlation coefficient $\left(\rho_{\mathrm{pe}}\right)$ and Spearman's rank-order correlation $\left(\rho_{\mathrm{sp}}\right)$ coefficient on ranked data, (Figueira et al. 2016) with $\rho$ : correlation coefficient and its standard deviation and 95\% highest posterior density (HPD) Credible Interval.

\begin{tabular}{|c|c|c|c|c|c|}
\hline \multirow{2}{*}{ Time interval with TESS } & \multicolumn{2}{|c|}{$\rho$} & \multicolumn{2}{|c|}{$95 \%$} & \multirow{2}{*}{ Sample size } \\
\hline & $\rho_{\mathrm{pe}}$ & $\rho_{\mathrm{sp}}$ & $\rho_{\mathrm{pe}}$ & $\rho_{\mathrm{sp}}$ & \\
\hline Year 1 & $0.989 \pm 0.005$ & $0.550 \pm 0.133$ & {$\left[\begin{array}{lll}0.979 & 0.996\end{array}\right]$} & {$\left[\begin{array}{lll}0.287 & 0.791\end{array}\right]$} & 24 \\
\hline Year 2 & $0.848 \pm 0.033$ & $0.394 \pm 0.099$ & {$\left[\begin{array}{lll}0.782 & 0.908\end{array}\right]$} & {$\left[\begin{array}{ll}0.196 & 0.578\end{array}\right]$} & 70 \\
\hline Year 3 & $0.520 \pm 0.071$ & $0.286 \pm 0.087$ & {$\left[\begin{array}{lll}0.384 & 0.656\end{array}\right]$} & {$\left[\begin{array}{lll}0.120 & 0.456\end{array}\right]$} & 104 \\
\hline Year 4 & $0.331 \pm 0.109$ & $0.265 \pm 0.113$ & {$\left[\begin{array}{ll}0.111 & 0.531\end{array}\right]$} & {$\left[\begin{array}{ll}0.043 & 0.486\end{array}\right]$} & 63 \\
\hline Whole sample & $0.812 \pm 0.026$ & $0.444 \pm 0.061$ & {$\left[\begin{array}{lll}0.764 & 0.862\end{array}\right]$} & {$\left[\begin{array}{ll}0.319 & 0.555\end{array}\right]$} & 171 \\
\hline
\end{tabular}

coefficient $\rho_{\mathrm{pe}}=0.989 \pm 0.005\left(\rho_{\mathrm{sp}}=0.550 \pm 0.133\right)$ for the correlation between photometric variability and RV jitter. As the time lag between RV measurement and TESS observation increases (subsample year 2, year 3 and year 4), the correlation becomes less strong and less significant. In the Table. 1, we presented the values of the $\rho_{\mathrm{pe}}$ and $\rho_{\mathrm{sp}}$ (Figueira et al. 2016). We also calculated the standard deviation and 95\% Highest Posterior Density (HPD) Credible Interval as described in Figueira et al. (2016) for the both $\rho_{\mathrm{pe}}$ and $\rho_{\mathrm{sp}}$ values. As can be read on Table 1 and Fig. 6, the correlation coefficients decrease to $\rho_{\mathrm{pe}}=0.848 \pm 0.033\left(\rho_{\mathrm{sp}}=0.394 \pm 0.099\right)$ for year 2 data, down to $\rho=0.520 \pm 0.071\left(\rho_{\mathrm{sp}}=0.286 \pm 0.087\right)$ and $\rho_{\mathrm{pe}}=0.331 \pm 0.109$ $\left(\rho_{\mathrm{sp}}=0.256 \pm 0.113\right)$ for year 3 and year 4 data, respectively. $\rho_{\mathrm{pe}}$ is always larger than $\rho_{\text {sp }}$ because of the extreme values in the subsets.

Therefore, our result shows that a correlation exists and decreases significantly when the time interval between the RV observations and photometric observation increases. This can 


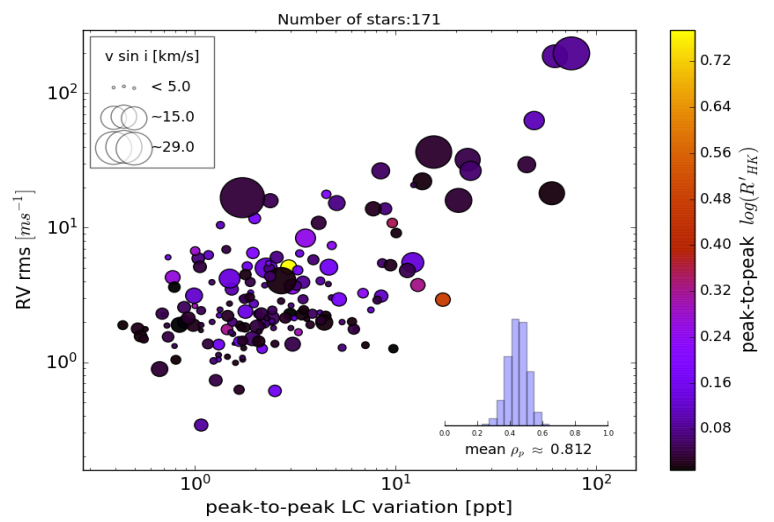

Fig. 7. Correlation between RV-RMS and peak-to-peak of light curve variation using the all years RV sampling (whole sample). Size of the points indicate the $v \sin i$ and color represent and peak-to-peak of $\log \left(R_{\mathrm{HK}}^{\prime}\right)$ within the RV measurements. The points above $6 \mathrm{ppt}$ have larger marker sizes and they filled up more space, thus this might cause difficulties in clearly recognizing the double law feature.

be interpreted as a consequence of the magnetic activity cycles which lead to departure from periodic variability to a quasiperiodic variability, and thus result in weakening the correlation over time. This has an impact on the predictive ability of using one dataset to estimate the other: since several studies aimed at estimating the RV jitter using photometric measurements (e.g., Oshagh et al. 2017; Bastien et al. 2014), here we note that such a prediction after more than $2 \mathrm{yr}$ of photometric observation can be imprecise since the correlation between RV jitter and peakto-peak light curve variability decreases $\left(\rho_{\mathrm{pe}} \sim 0.8\right.$ at year 2 to $\rho_{\mathrm{pe}} \sim 0.5$ at year 3 ).

\subsection{Overall correlation using the whole sample}

The overall correlation between RV-RMS and peak-to-peak of light curve for the whole sample within the whole $4 \mathrm{yr}$ is presented in Fig. 7. The correlation is significant; The mean value of $\rho_{\mathrm{pe}}$ is $0.812 \pm 0.026$ and that of $\rho_{\mathrm{sp}}$ is $0.444 \pm 0.061$. In this comparison it is important to mention that while the RV jitter is derived using relatively long-term observations (see Fig. B.4), the photometric peak-to-peak is measured during TESS observations, of roughly one month.

Functional form of the dependence. Careful inspection of the shape of the RV-RMS vs. peak-to-peak photometry dependence in Fig. 7 (see also Fig. 6, especially the year 2 subplot), shows hints of a double behavior, a region where the dependence is weak or plateau (peak-to-peak of light curve variation $<6 \mathrm{ppt}$ ) and a region where the dependence is steeper, being associated to a stronger correlation coefficient (peak-to-peak of light curve variation $>6 \mathrm{ppt})$.

Here we aim to examine different functional forms that can properly describe the whole sample (Fig. 7) and individual subsamples (Fig. 6). To test this, we decided to fit RV jitter and photometric peak-to-peak variability using two types of functional models, a) a power law function and b) piecewise power law function (which is composed of two power law functions with a knee). We used Markov-chain Monte Carlo (MCMC) sampling using the library emcee (Foreman-Mackey et al. 2013) to perform the fit and obtain the uncertainties of the fitted parameters; for details refer to Appendix A. The best fits are overplotted in Fig. 8 for year 2 which is closest subsampling group to the TESS observations with fairly sufficient number of data points. The best fits are also overplotted in Fig. 9 for the all
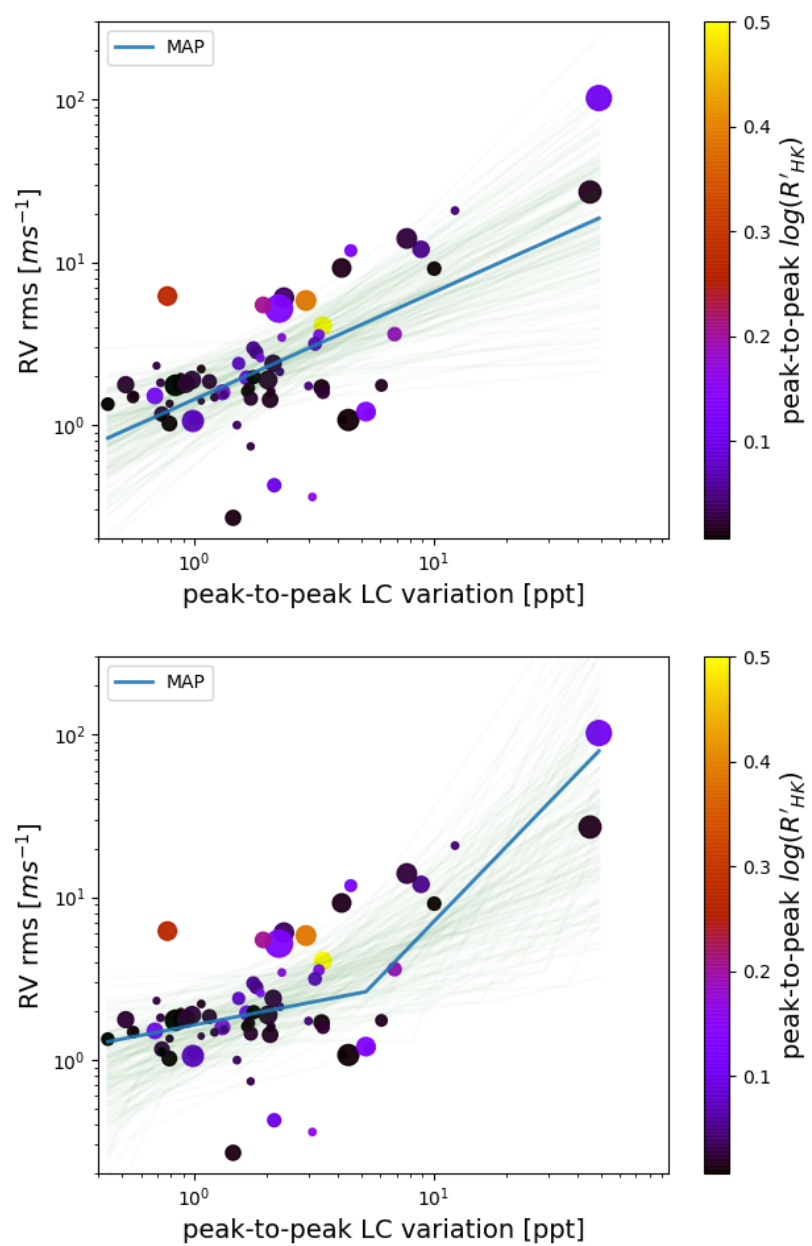

Fig. 8. Year 2 sample with single power law function fit (top plot) and a piecewise function with two power law function fit (bottom plot); In both panels the best fit indicated with the MAP.

RV sampling. All fitted parameters are reported in the Table. A.1. The best fit indicated with the maximum a posteriori (MAP).

We compare the best fitted models using RMS of the residual $\left(\mathrm{RMS}_{\mathrm{resi}}\right)$ and Bayesian information criterion (BIC). For all the subsamples and also for the whole sample the BIC values are similar between two models. However, since the piecewise function is penalized for the two extra parameters, BIC values for the single power law function has lower BIC than piecewise function, which suggests that the single power law function as a better model, but with a low significant evidence. We considered BIC difference to be significant if $\triangle \mathrm{BIC}>10$ (Liddle 2007; Arevalo et al. 2017). On the other hand, for the subsample and also for the whole sample, the fits using piecewise model have lower $\mathrm{RMS}_{\text {resi }}$, which means it can describe the data more precisely. For instance for the whole sample $\left(\mathrm{RMS}_{\mathrm{resi}} \sim 0.35\right.$ vs. 0.29 ) for single and piecewise model, respectively. If we consider the piecewise function as the best model for describing the data, the knee is located at a value of $\sim 6.5$ ppt peak-to-peak of light curve variation.

\section{Correlations with activity level and rotational period}

The large data-set presented here also allows us to explore other correlations that may be relevant for the estimation of RV jitter in different stars. 

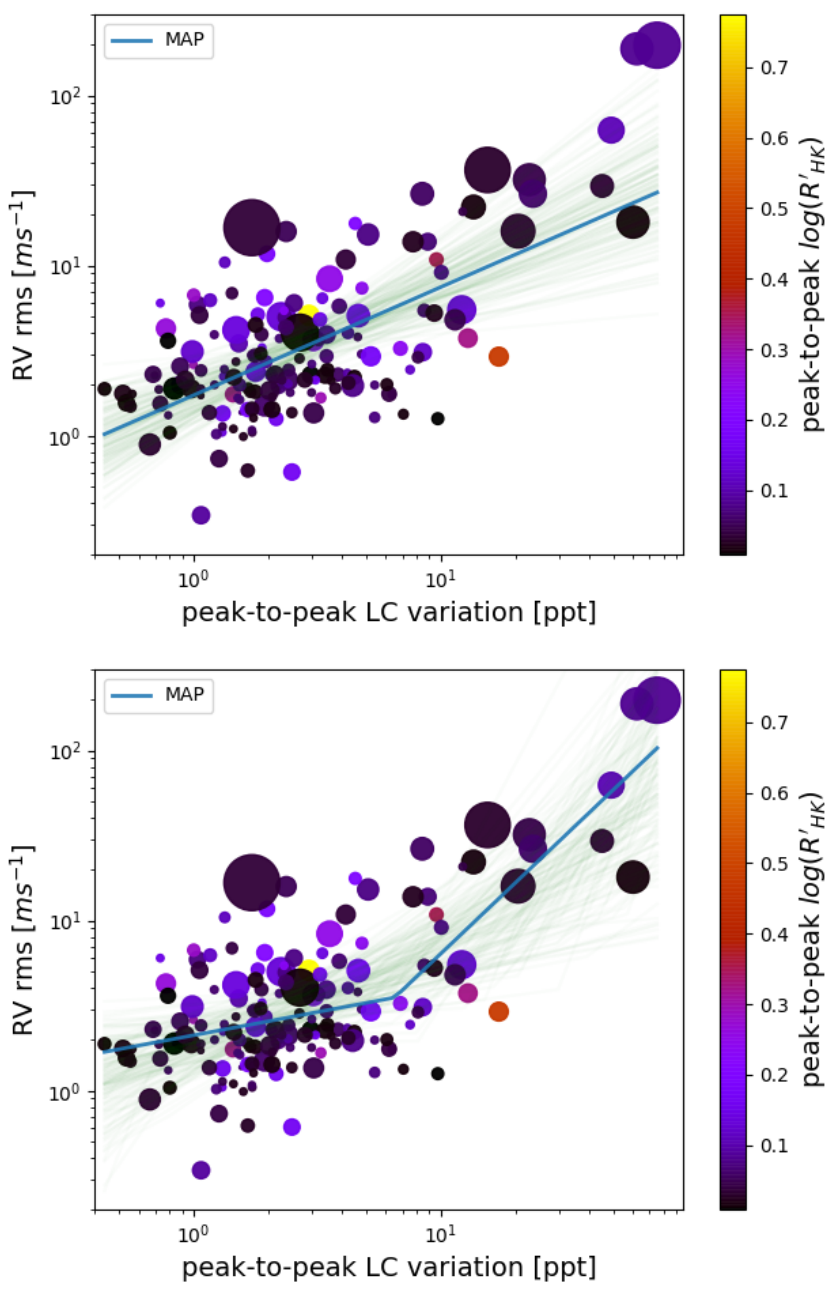

Fig. 9. Top plot: same with single power law function fit. Bottom plot: all RV sampling group with two piecewise power law fitting. In both panels the best fit indicated with the MAP.

\subsection{Correlation with $\log \left(R_{H K}^{\prime}\right)$}

In the Figs. 10 and 11, we present the mean $\log \left(R_{\mathrm{HK}}^{\prime}\right)$ values versus RV-RMS and peak-to-peak photometric variability for the whole sample. We also fitted the power law function and use MCMC to find the error in order to provide a relationship between the RV-RMS, peak-to-peak variability and $\log \left(R_{\mathrm{HK}}^{\prime}\right)$ given by:

$\mathrm{RV}_{\mathrm{RMS}} \propto\left(R_{\mathrm{HK}}^{\prime}\right)^{a}, \mathrm{a}=1.382_{-0.344}^{0.341}$.

Our result is compatible with Saar et al. (2003) which reported $a=1.1$ however less compatible with Santos et al. (2000) which reported $a=0.55$. We obtained using the sample of stars in this project. If we assume that the same functional dependence exists between the $\log \left(R_{\mathrm{HK}}^{\prime}\right)$ and peak-to-peak photometric we can write it as

$\mathrm{LC}_{\mathrm{pp}} \propto\left(R_{\mathrm{HK}}^{\prime}\right)^{a}, \mathrm{a}=1.304_{-0.335}^{0.338}$,

where $\mathrm{LC}_{\mathrm{pp}}$ is peak-to-peak of light curve variation.

\subsection{Correlation with stellar rotation period}

In Top panel in Fig. 12, we present RV-RMS and the peak-topeak light curve variation for the subsample of 71 stars with

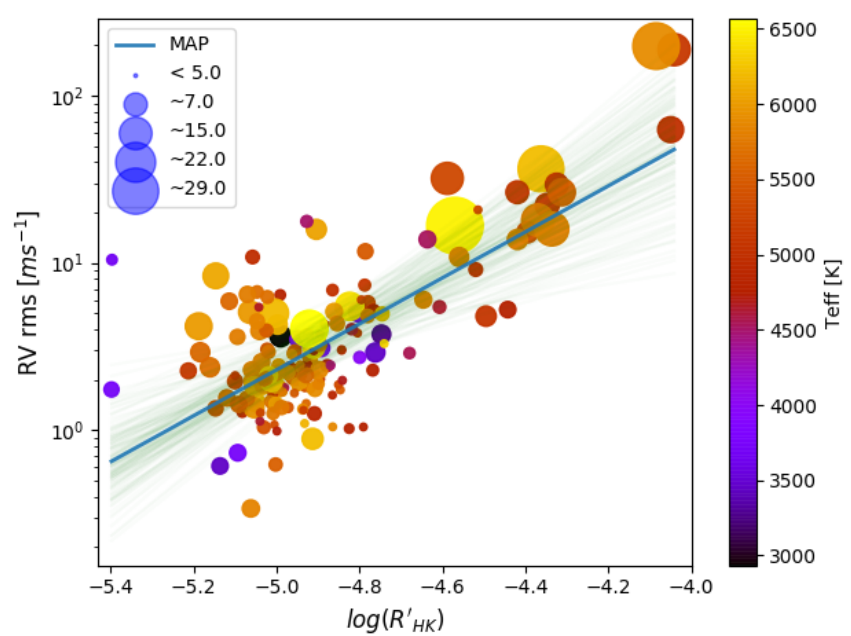

Fig. 10. $\log \left(R_{\mathrm{HK}}^{\prime}\right)$ vs. RV-RMS of light curve with the best fit. The same method in MCMC analysis explained in Appendix A, $\log 10(y)=a x+$ $b: a=1.382_{-0.344}^{0.341}, b=7.271_{-1.683}^{1.670}$.

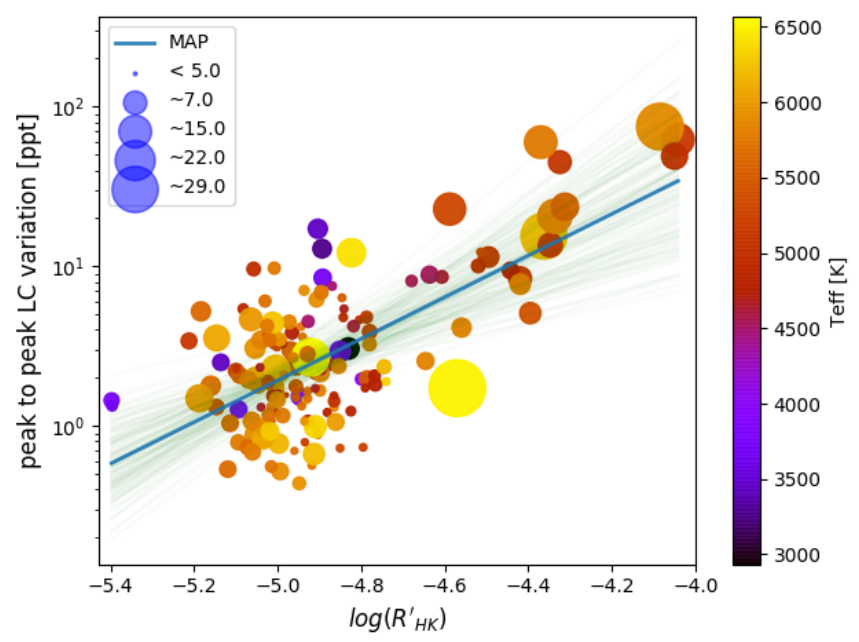

Fig. 11. $\log \left(R_{\mathrm{HK}}^{\prime}\right)$ vs. peak-to-peak of light curve variation with the best fit. The same method in MCMC analysis explained in Appendix A, $\log 10(y)=a x+b: a=1.304_{-0.335}^{0.338}, b=6.804_{-1.641}^{1.659}$.

measured rotation periods (coded with marker size). The color bar represents the effective temperature. One can easily notice that stars with large RV-RMS and a large peak-to-peak photometric variability are mostly fast rotating stars (less than 13 days) and there is a hint of temperature dependency. Bottom panel in Fig. 12 shows the same 71 stars but color bar indicates the rotation period value, and circle size the $v \sin i$ obtained spectroscopically, which again confirms the previous result we found.

In Fig. 13, we present a similar plot to Fig. 12 but this time for 29 stars where we could identify faculae or spot dominated patterns using the method described in Sect. 3.2. We found 9 faculae dominated stars (which were also slow rotators as is expected for faculae dominated stars), and 20 spot-dominated stars. We show faculae and spot dominated stars in yellow and black, respectively. In this sample, 20 stars can be classified as fast rotators (rotation period $<15$ days), and large fraction of them (13 out 20$)$ are spot dominated. This result is in strong agreement with Montet et al. (2017), where they reported 15 days as the threshold in rotation period for separating spot-faculae dominated regimes. Moreover, faculae-dominated stars tend to have 

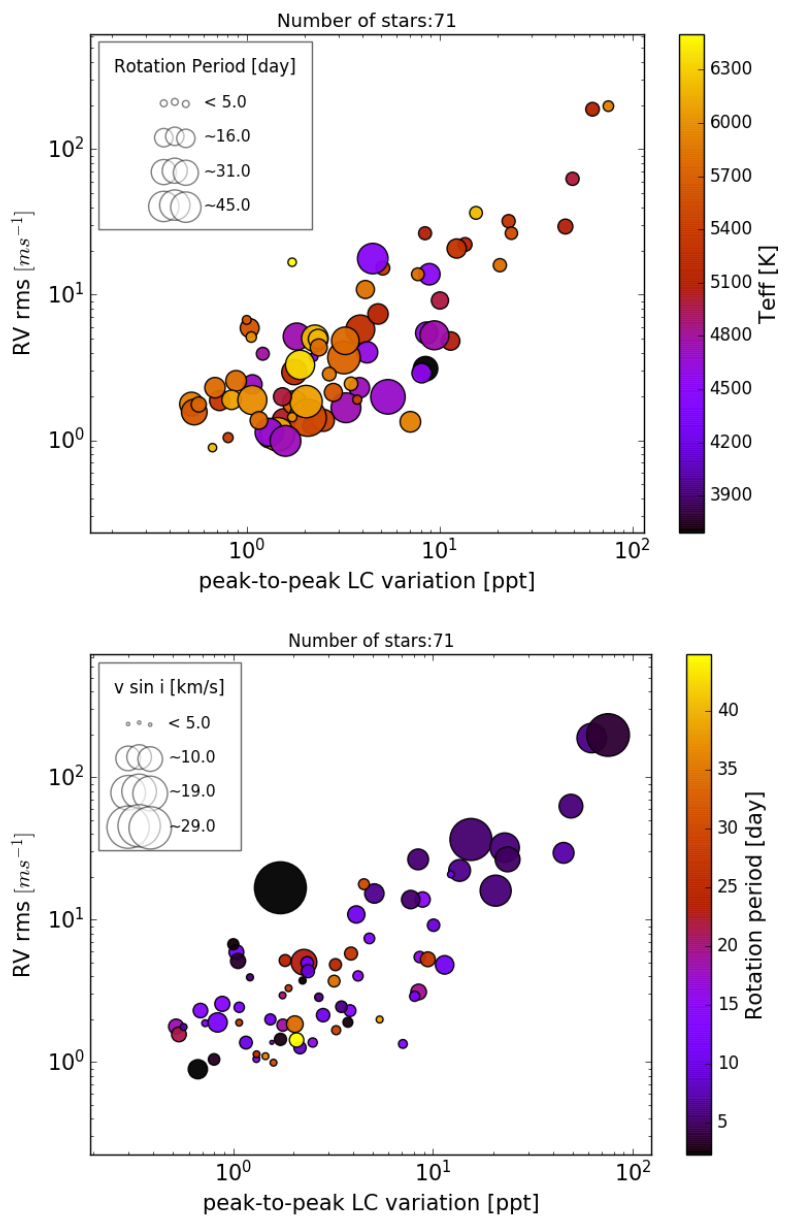

Fig. 12. RV-RMS and peak-to-peak of light curve variation for the subsample of 71 stars. Top plot: circle sizes represents the value of rotation period found by GPS method, see Table. C.1. Color bar indicates the stellar effective temperature. Bottom plot: similar than top panel but, color bar indicates the rotation period value, and circle size the $v \sin i$ obtained spectroscopically.

low photometric peak-to-peak variability, due to the low contrast of faculae's region, and therefore are mostly below the $6.5 \mathrm{ppt}$ limit. Thus, the $6.5 \mathrm{ppt}$ limit can be also interpreted as the photometric variability transition between the spot-dominated and the faculae-dominated regime. However, the sample of 29 stars is too small to generalize.

\section{Application of our result on the TESS planet candidates' host stars}

It is difficult to define the criteria for the most suitable targets for RV follow up of transiting planet candidates. In functional form of the dependence we saw that the dependence of RV jitter on photometric peak-to-peak variation shows a hint of knee at $6.5 \mathrm{ppt}$. We use this value as an upper threshold to identify the targets on the TESS object of interest (TOI) catalog ${ }^{13}$ with the lowest RV jitter. We selected TOI catalog targets with publicly available light curves in the archive. Since we are mostly interested in the cooler stars, we selected the stars with $T_{\text {eff }}$ lower than $6800 \mathrm{~K}$. Although in our sample we have filtered out the evolved stars already, we selected the stars with the radius less than $2.0 R_{\text {sun }}$ to narrow our analysis and select stars similar to our

\footnotetext{
${ }^{13}$ https://tess.mit.edu/toi-releases/
}

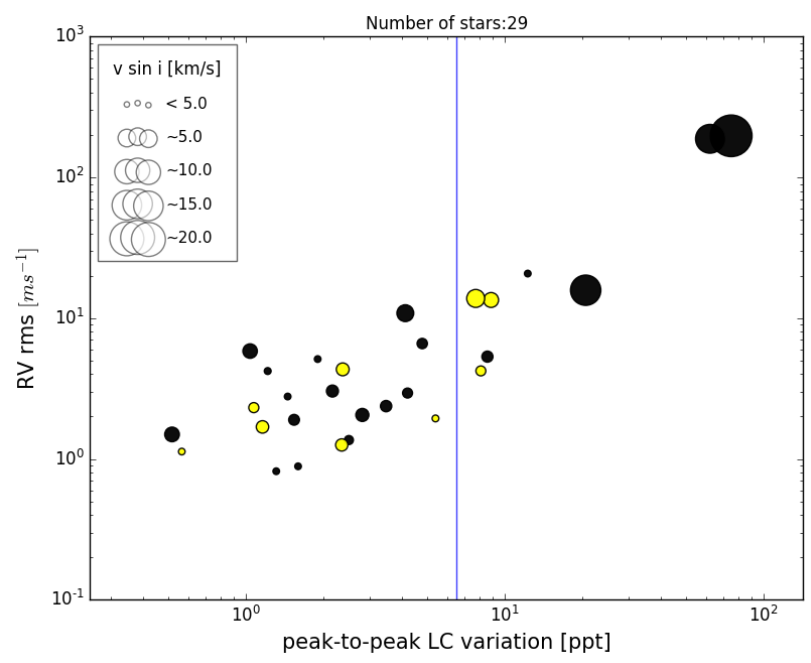

Fig. 13. RV-RMS and the peak-to-peak of light curve variation for the subsample of 20 stars with spot dominance and 9 stars with faculae dominance in their light curves. Circle sizes represents the $v \sin i$ value determined spectroscopically. Black color indicates spot dominated and yellow indicates faculae dominated. The Blue vertical line shows the knee point presented in Fig. 9 in peak-to-peak light curve variation at $6.5 \mathrm{ppt}$.

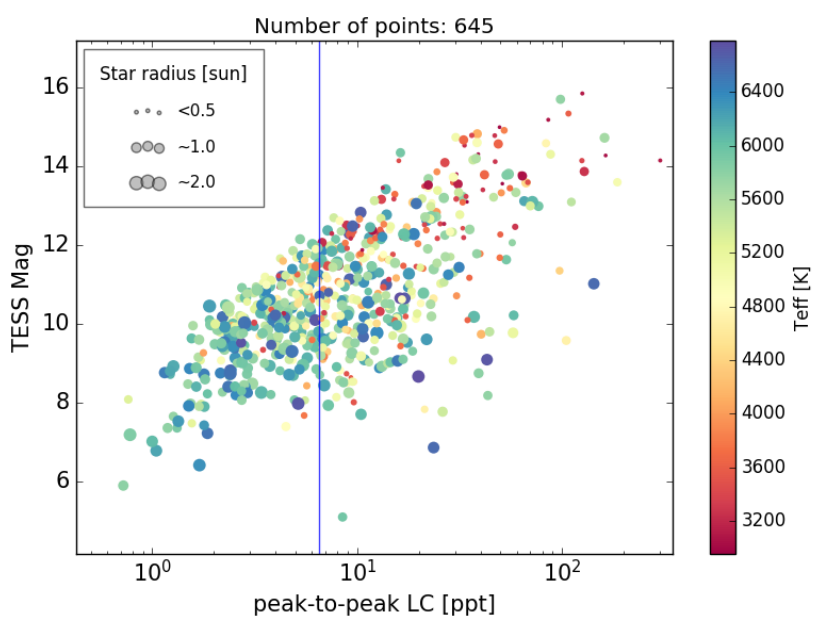

Fig. 14. Peak-to-peak of light curve versus TESS mag. Color bar shows the stellar effective temperature and size shows the stellar radius. The Blue vertical line shows the knee point presented in Fig. 9 in peak-topeak light curve variation at $6.5 \mathrm{ppt}$.

the Sun. In this sample, we used $T_{\text {eff }}, R_{\text {sun }}$ and TESS magnitudes as reported in the TIC (Stassun et al. 2018).

We then removed all the flux points within the transit reported by the TOI catalog. After eliminating the transit lightcurves, we applied the same procedures explained in Sect. 2.2 and estimated the peak-to-peak flux variation for each TOI host star.

In the Fig. 14, we present the results of the peak-to-peak variation versus TESS magnitude. Stars with different $R_{\text {sun }}$ values are denoted by points with different sizes. The color denotes the effective temperature of the star. The vertical line shows where we believe a critical knee point in Fig. 9 is. The amplitude of RV jitter above this limit of photometric variability $(6.5 \mathrm{ppt})$ can dramatically increase. Forty-three percent of the stars in this sample have light curve peak-to-peak variations less than $6.5 \mathrm{ppt}$ (279 out of 645 stars). Mean, median, and interquartile 
range of RV-RMS of the stars with photometric peak-to-peak variability below $6.5 \mathrm{ppt}$ in our sample are $3.62 \mathrm{~ms}^{-1}, 2.77 \mathrm{~ms}^{-1}$, and $2.54 \mathrm{~ms}^{-1}$, respectively. We note that in this figure we can also clearly see a correlation between the TESS magnitude and peak-to-peak variation as well as $T_{\text {eff }}$, which can be part of the instrumental as well as the astrophysical effects. The correlation of the peak-to-peak light curve variation with star magnitudes can be explained by the TESS photon noise (see more Fig. 8 in Ricker et al. 2014b). We tested the impact of photon noise by seeking for any correlation between the RV-RMS and stars' magnitude, as well as between the photometric peak-to-peak variation and stars' magnitude in our sample, and could not find any significant correlation.

\section{Discussion and conclusion}

We present a study on the correlation between RV jitter and photometric variability. We used a sample of 171 starts that have been observed by both the HARPS spectrograph and TESS space telescope. We derived the RV-RMS and peak-to-peak of light curve variability as well as the stellar parameters.

We found a strong correlation between the RV jitter and photometric flux variability. We divided our sample into four subsamples in order to investigate the impact of time interval between RV measurements and light curves. We found that the correlation becomes weaker as the time lags between the RV measurements and light curves increase. Within year 1 and year 2 , the correlation is strong but the year 3 and year 4 show relatively weaker correlation. We can conclude the maximum time difference in which the light curve peak-to-peak can be used to predict the RV jitter is two years. After that the information content of photometric viability is lost, probably the consequence of the evolution of the stellar magnetic cycle. This could imply that majority of these stars with different spectral types should have a cycle shorter than $6 \mathrm{yr}$, assuming that half an activity cycle is sufficient to bring the observables out of phase and diminishes the correlation (for instance if the RVs were taken at activity maximum and photometry were obtained at activity minimum or vice-versa).

We also examined different functional forms which could represent better the observed correlations. We compare the best fitted model using $\mathrm{RMS}_{\text {resi }}$ and BIC. For the whole sample the fit of piecewise power law functions, with a knee at $6.5 \mathrm{ppt}$ in peak-to-peak of light curve variation, describes the data more precisely with lower $\mathrm{RMS}_{\text {resi }}$ without considering the cost of the two extra parameters. We also fitted the two models on the year 1, year 2, year 3, and year 4 subsampling (see Table A.1). The results show that for the year 2 , which is the closest subsampling group to the TESS observations with fairly sufficient number of data points as well as a strong correlation, the piecewise power law also describes the data better than the single power law with lower $\mathrm{RMS}_{\text {resi }}$, however, the BIC is larger for the piecewise model because of the two extra parameters in this model. The correlation between RV-RMS and peak-to-peak of light curve variability of showed two regions separating with a knee point where the strength of the correlation changes. This might be due to the transition from the spot-dominated regime to the faculae-dominated regime. These two regimes have already been discussed in several studies (Lockwood et al. 1997; Radick et al. 1998; Shapiro et al. 2016, 2020; Oshagh et al. 2017)

Meunier \& Lagrange (2019) and Meunier et al. (2019) simulated the RVs and photometric time series and derived the flux variability as well as the global RV jitter for F to early $\mathrm{K}$ stars. They showed that there is a relationship between the photometric variability and RV jitter considering the dependency of stellar parameters such as rotation period and $T_{\text {eff }}$ as well as inclination. These simulations showed presence of a knee point in the correlation between photometric variability and RV jitter, however, the knee point and also the range of values are in a different domain than our findings. There could be several reason for these disagreements such as: (a) our observational sample of stars could have more complex and random spot or faculae than the ones simulated in Meunier \& Lagrange (2019). (b) our observational RV sample were not simultaneous with the TESS light curve, while in Meunier \& Lagrange (2019) the simulation were done for simultaneous observations. (c) the observation sample could have unknown companions which can produce RV variation in time series. However, the dispersion of the simulations for RV-RMS and photometric variability (see Fig. 9 in Meunier \& Lagrange 2019) are compatible with the observed dispersion of the whole sample.

We also found that there is a strong correlation between the chromospheric activity index $\left(\log \left(R_{\mathrm{HK}}^{\prime}\right)\right)$ and RV jitter and also with photometric variability, which are both in agreement with previous studies (Santos et al. 2000; Wright 2005; Saar et al. 2003; Meunier et al. 2019). Also the observed correlation between $\log \left(R_{\mathrm{HK}}^{\prime}\right)$ and peak-to-peak of photometric variation is compatible to the simulation's result within the range $-5.0<$ $\log \left(R_{\mathrm{HK}}^{\prime}\right)<-4.6$ (see Fig. 5 in Meunier \& Lagrange 2019). We provided an updated version of the relation between $\log \left(R_{\mathrm{HK}}^{\prime}\right)$ and RV jitter and also with peak-to-peak of light curve variability.

We were able to estimate the rotation period of 71 stars, out of 171 stars in our sample, using the TESS light curve. Then we investigated the effect of this parameter on the correlation between RV-RMS and peak-to-peak of light curve variability. Our result demonstrated that slow rotating stars (which are the ones also we found to be faculae dominated) create lower RV jitter as well as lower peak-to-peak photometric variability, and on the other hand fast rotating star, $P_{\text {rot }} \leq 5$ day (which are the ones also we found to be spot dominated) generate much larger RV jitter and photometric variability.

We have also looked into the correlation of the other parameters which might affect the peak-to-peak of light curve vs RV-RMS correlation such as $T_{\text {eff }},[\mathrm{Fe} / \mathrm{H}]$ and the number of nights that the star has been observed in RV. We could not find any significant trend caused by these parameters on the peak-topeak of light curve vs. RV-RMS correlation (Appendix B). We also examined our results by using just the first sector for the stars which have more than one TESS sector observations and we did not see any significant changes in the results. Finally, we attempted to select the best targets, in terms of the lowest RV jitter, in TESS Objects of Interest catalog. To do that, we used our estimated knee point at $6.5 \mathrm{ppt}$ in photometric variability. We estimated that $42 \%$ of the stars in a specific sample of the TOI catalog have the peak-to-peak photometric variability of less than $6.5 \mathrm{ppt}$ and, therefore, will have a small amplitude RV jitter (less than $\sim 3 \mathrm{~ms}^{-1}$ ) which can be essential for the confirmation of Earth-mass exoplanets at short orbits around low-mass stars in TESS objects of interest. (see Table. C.2).

Acknowledgements. We would like to thank the anonymous referee for insightful and constructive comments, which added significantly to the clarity of this paper. This work was supported by FCT - Fundação para a Ciência e a Tecnologia through national funds and by FEDER through COMPETE2020 Programa Operacional Competitividade e Internacionalização by these grants: UID/FIS/04434/2019; UIDB/04434/2020 \& UIDP/04434/2020; PTDC/ FIS-AST/32113/2017 \& POCI-01-0145-FEDER-032113; PTDC/FIS-AST/ 28953/2017 \& POCI-01-0145-FEDER-028953. S.H. and B.A. acknowledge support by the fellowships PD/BD/128119/2016 and PD/BD/135226/2017 
funded by FCT (Portugal) and POCH/FSE (EC). S.S., V.A., O.D.S.D and J.P.F. acknowledge support from FCT through work contracts $\mathrm{n} \mathrm{O}_{\mathrm{S}}$ IF/00028/2014/CP1215/CT0002, IF/00650/2015/CP1273, DL 57/2016/ CP1364/CT0004, DL 57/2016/CP1364/CT0005. M.O. acknowledges the support of the Deutsche Forschungsgemeinschft (DFG) priority program SPP 1992 "Exploring the Diversity of Extrasolar Planets (RE 1664/17-1)" M.O., E.A.G also acknowledge the support of the FCT/DAAD bilateral grant 2019 (DAAD ID: 57453096). M.O. acknowledges research funding from the Deutsche Forschungsgemeinschft (DFG, German Research Foundation) - OS 508/1-1. This research made use of Astropy, a community-developed core Python package for Astronomy (Astropy Collaboration 2013, 2018), and the NumPy, SciPy, Matplotlib, tesscut, celerite Python modules (Van Der Walt et al 2011; Jones et al. 2001-; Hunter 2007; Brasseur et al. 2019; Foreman-Mackey et al. 2017) and pandas (McKinney 2010, 2011). This research made use of Lightkurve, a Python package for Kepler and TESS data analysis (Lightkurve Collaboration 2018). This paper includes data collected with the TESS mission, obtained from the MAST data archive at the Space Telescope Science Institute (STScI). Funding for the TESS mission is provided by the NASA Explorer Program. STScI is operated by the Association of Universities for Research in Astronomy, Inc., under NASA contract NAS 5-26555. This work is based on observations collected at the European Organization for Astronomical Research in the Southern Hemisphere under ESO programs: '0100.C-0097(A)','0100.C0487(A)','0100.C-0836(A)','0100.D-0444(A)', '0101.C-0379(A)','0101.D-0494 (A)','0101.D-0494(B)','060.A-9036(A)','089.C-0732(A)',090.C-0421(A)','093. C-0062(A)','095.C-0040(A)','095.C-0551(A)','095.C-0799', '095.C-0799(A)', '096.C-0053(A)','096.C-0460(A)','096.C-0499(A)', '096.C-0876(A)', '097.C0021(A)',097.C-0090(A)', '097.C-0390(B)', '097.C-0571(A)', '098-C-0518(A)', '098.C-0269(A)', '098.C-0269(B)', '098.C-0366(A)', '098.C-0518(A)','098.C 0739(A)','099.C-0205(A)','099.C-0458(A)',099.C-0798(A)','183.C-0437(A)', '188.C-0265(O)', '188.C-0265(P)', '188.C-0265(Q)','188.C-0265(R)', '190.C0027(A)','191.C-0873(A)','192.C-0224','192.C-0224(C)','192.C-0852(A)','196. C-0042','196.C-0042(D)','196.C-0042(E)', '196.C-1006(A)','198.C-0836(A)', '198.C-0838(A)','60.A-9036(A)','Lagrange'.

\section{References}

Adibekyan, V., Sousa, S. G., \& Santos, N. C. 2018, in Asteroseismology and Exoplanets: Listening to the Stars and Searching for New Worlds, eds. T. L. Campante, N. C. Santos, \& M. J. P. F. G. Monteiro (Berlin: Springer), 49, 225 Aigrain, S., Pont, F., \& Zucker, S. 2012, MNRAS, 419, 3147

Amazo-Gómez, E. M., Shapiro, A. I., Solanki, S. K., et al. 2020, A\&A 636, A69

Arevalo, F., Cid, A., \& Moya, J. 2017, Eur. Phys. J. C, 77, 565

Astropy Collaboration (Robitaille, T. P., et al.) 2013, A\&A, 558, A33

Astropy Collaboration (Price-Whelan, A. M., et al.) 2018, AJ, 156, 123

Barros, S. C. C., Almenara, J. M., Deleuil, M., et al. 2014, A\&A, 569, A74

Bastien, F. A., Stassun, K. G., Basri, G., \& Pepper, J. 2013, Nature, 500, 427

Bastien, F. A., Stassun, K. G., Pepper, J., et al. 2014, AJ, 147, 29

Boisse, I., Bouchy, F., Hébrard, G., et al. 2011a, IAU Symp., 273, 281

Boisse, I., Bouchy, F., Hébrard, G., et al. 2011b, A\&A, 528, A4

Borucki, W. J., Koch, D., Basri, G., et al. 2010, Science, 327, 977

Brasseur, C. E., Phillip, C., Fleming, S. W., Mullally, S. E., \& White, R. L. 2019 Astrophysics Source Code Library [record ascl : 1905.007]

Bryson, S. T., Jenkins, J. M., Klaus, T. C., et al. 2017, Kepler Data Processing Handbook: Target and Aperture Definitions: Selecting Pixels for Kepler Downlink, Tech. rep.

Cegla, H. M., Stassun, K. G., Watson, C. A., Bastien, F. A., \& Pepper, J. 2014, ApJ, 780, 104

Cloutier, R., Doyon, R., Bouchy, F., \& Hébrard, G. 2018, AJ, 156, 82

Czesla, S., Huber, K. F., Wolter, U., Schröter, S., \& Schmitt, J. H. M. M. 2009, A\&A, 505, 1277

Desort, M., Lagrange, A. M., Galland, F., Udry, S., \& Mayor, M. 2007, A\&A, 473, 983

Díaz, R. F., Ségransan, D., Udry, S., et al. 2016, A\&A, 585, A134

Dumusque, X., Udry, S., Lovis, C., Santos, N. C., \& Monteiro, M. J. P. F. G. 2011a, A\&A, 525, A140

Dumusque, X., Santos, N. C., Udry, S., Lovis, C., \& Bonfils, X. 2011b, A\&A, 527, A82

Duncan, D. K., Vaughan, A. H., Wilson, O. C., et al. 1991, ApJS, 76, 383

Figueira, P., Marmier, M., Bonfils, X., et al. 2010, A\&A, 513, L8

Figueira, P., Faria, J. P., Adibekyan, V. Z., Oshagh, M., \& Santos, N. C. 2016, Orig. Life Evol. Biosph., 46, 385

Findeisen, K., Hillenbrand, L., \& Soderblom, D. 2011, AJ, 142, 23

Fischer, D. A., \& Valenti, J. 2005, ApJ, 622, 1102

Fischer, D. A., Anglada-Escude, G., Arriagada, P., et al. 2016, PASP, 128, 066001

Foreman-Mackey, D., Hogg, D. W., Lang, D., \& Goodman, J. 2013, PASP, 125, 306

Foreman-Mackey, D., Agol, E., Angus, R., \& Ambikasaran, S. 2017, AJ, 154, 220
Gomes da Silva, J., Santos, N. C., Bonfils, X., et al. 2011, A\&A, 534, A30 Gomes da Silva, J., Figueira, P., Santos, N., \& Faria, J. 2018, J. Open Source Softw., 3, 667

Hojjatpanah, S., Figueira, P., Santos, N. C., et al. 2019, A\&A, 629, A80

Huélamo, N., Figueira, P., Bonfils, X., et al. 2008, A\&A, 489, L9

Hunter, J. D. 2007, Comput. Sci. Eng., 9, 90

Jones, E., Oliphant, T., Peterson, P., et al. 2001-, SciPy: Open source scientific tools for Python

Korhonen, H., Andersen, J. M., Piskunov, N., et al. 2015, MNRAS, 448, 3038

Liddle, A. R. 2007, MNRAS, 377, L74

Lightkurve Collaboration (Cardoso, J. V. d. M., et al.) 2018, Astrophysics Source Code Library [record ascl: 1812.013 ]

Lo Curto, G., Pepe, F., Avila, G., et al. 2015, The Messenger, 162, 9

Lockwood, G. W., Skiff, B. A., \& Radick, R. R. 1997, ApJ, 485, 789

Maldonado, J., Scandariato, G., Stelzer, B., et al. 2017, A\&A, 598, A27

Mamajek, E. E., \& Hillenbrand, L. A. 2008, ApJ, 687, 1264

Martin, D. C., Fanson, J., Schiminovich, D., et al. 2005, ApJ, 619, L1

Mayor, M., Pepe, F., Queloz, D., et al. 2003, The Messenger, 114, 20

Mayor, M., Udry, S., Naef, D., et al. 2004, A\&A, 415, 391

McKinney, W. 2010, in Proceedings of the 9th Python in Science Conference, Vol. 445, Austin, TX, 51-56

McKinney, W. 2011, Python for High Performance and Scientific Computing, 14

Meunier, N., \& Lagrange, A. M. 2019, A\&A, 629, A42

Meunier, N., Lagrange, A. M., \& Cuzacq, S. 2019, A\&A, 632, A81

Montet, B. T., Tovar, G., \& Foreman-Mackey, D. 2017, ApJ, 851, 116

Morris, R. L., Twicken, J. D., Smith, J. C., et al. 2017, Kepler Data Processing Handbook: Photometric Analysis, Tech. rep.

Neves, V., Bonfils, X., Santos, N., et al. 2014, A\&A, 568, A121

Nielsen, M. B., Gizon, L., Schunker, H., \& Karoff, C. 2013, A\&A, 557, L10

Noyes, R. W., Hartmann, L. W., Baliunas, S. L., Duncan, D. K., \& Vaughan, A. H. 1984, ApJ, 279, 763

Oshagh, M. 2018, in Asteroseismology and Exoplanets: Listening to the Stars and Searching for New Worlds (Berlin: Springer), Vol. 49, 239

Oshagh, M., Boisse, I., Boué, G., et al. 2013a, A\&A, 549, A35

Oshagh, M., Santos, N. C., Boisse, I., et al. 2013b, A\&A, 556, A19

Oshagh, M., Santos, N. C., Boisse, I., et al. 2015, Euro. Phys. J. Web Conf., 101, 05003

Oshagh, M., Santos, N. C., Figueira, P., et al. 2017, A\&A, 606, A107

Pepe, F., Molaro, P., Cristiani, S., et al. 2014, Astron. Nachr., 335, 8

Queloz, D., Henry, G. W., Sivan, J. P., et al. 2001, A\&A, 379, 279

Radick, R. R., Lockwood, G. W., Skiff, B. A., \& Baliunas, S. L. 1998, ApJS, 118, 239

Rauer, H., Catala, C., Aerts, C., et al. 2014, Exp. Astron., 38, 249

Reffert, S., Bergmann, C., Quirrenbach, A., Trifonov, T., \& Künstler, A. 2015, A\&A, 574, A116

Reinhold, T., \& Hekker, S. 2020, A\&A, 635, A43

Ricker, G. R., Winn, J. N., Vanderspek, R., et al. 2014a, in Proc. SPIE Conf. Ser., 9143, 914320

Ricker, G. R., Winn, J. N., Vanderspek, R., et al. 2014b, J. Astron. Teles. Instrum. Systems, 1, 014003

Robertson, P., Mahadevan, S., Endl, M., \& Roy, A. 2014, Science, 345, 440

Saar, S. H., \& Donahue, R. A. 1997, ApJ, 485, 319

Saar, S. H., Butler, R. P., \& Marcy, G. W. 1998, ApJ, 498, L153

Saar, S. H., Hatzes, A., Cochran, W., \& Paulson, D. 2003, The Future of CoolStar Astrophysics: 12th Cambridge Workshop on Cool Stars, Stellar Systems, and the Sun, eds. A. Brown, G. M. Harper, \& T. R. Ayres (Cambridge: Cambridge University Press), 12. 694

Santos, N. C., Mayor, M., Naef, D., et al. 2000, A\&A, 361, 265

Santos, N. C., Mayor, M., Naef, D., et al. 2002, A\&A, 392, 215

Santos, N. C., Gomes da Silva, J., Lovis, C., \& Melo, C. 2010, A\&A, 511, A54

Santos, N. C., Mortier, A., Faria, J. P., et al. 2014, A\&A, 566, A35

Shapiro, A. I., Solanki, S. K., Krivova, N. A., Yeo, K. L., \& Schmutz, W. K. 2016, A\&A, 589, A46

Shapiro, A. I., Amazo-Gómez, E. M., Krivova, N. A., \& Solanki, S. K. 2020, A\&A, 633, A32

Sousa, S. G. 2014, Determination of Atmospheric Parameters of B-, A-, Fand G-Type Stars. Series: GeoPlanet: Earth and Planetary Sciences, eds. E., Niemczura, B., Smalley, \& W. Pych (Cham: Springer International Publishing), 297

Stassun, K. G., Oelkers, R. J., Pepper, J., et al. 2018, AJ, 156, 102

Tayar, J., Stassun, K. G., \& Corsaro, E. 2019, ApJ, 883, 195

Van Der Walt, S., Colbert, S. C., \& Varoquaux, G. 2011, Comput. Sci. Eng., 13, 22

Vaughan, A. H., Preston, G. W., \& Wilson, O. C. 1978, PASP, 90, 267

Vida, K., Oláh, K., Kővári, Z., et al. 2019, ApJ, 884, 160

Wright, J. T. 2005, PASP, 117, 657 


\section{Appendix A: Correlation and uncertainties estimation}

Table A.1. Maximum a posteriori (MAP) with error for parameters which described in the Eqs. (A.1) and (A.2) as well as the Bayesian information criterion (BIC) and RMS of the residual $\left(\mathrm{RMS}_{\text {resi }}\right)$ between the model and data for the two models: single power law function and piecewise power laws functions.

\begin{tabular}{|c|c|c|c|c|c|c|c|c|c|c|}
\hline & \multicolumn{6}{|c|}{ Posterior } & \multicolumn{2}{|c|}{$\mathrm{RMS}_{\mathrm{resi}}$} & \multicolumn{2}{|c|}{ BIC } \\
\hline & \multicolumn{2}{|c|}{ Single } & \multicolumn{4}{|c|}{ Piecewise } & \multirow{2}{*}{ Single } & \multirow{2}{*}{ Piecewise } & \multirow{2}{*}{ Single } & \multirow{2}{*}{ Piecewise } \\
\hline & $a$ & $b$ & $x_{0}$ & $y_{0}$ & $k_{1}$ & $k_{2}$ & & & & \\
\hline Year 1 & $0.825_{-0.399}^{0.417}$ & $0.038_{-0.225}^{0.233}$ & $0.610_{-0.788}^{0.573}$ & $0.338_{-0.470}^{0.664}$ & $0.392_{-0.458}^{0.635}$ & $1.450_{-0.833}^{1.396}$ & 0.30 & 0.25 & 8.6 & 14.3 \\
\hline Year 2 & $0.659_{-0.285}^{0.289}$ & $0.156_{-0.148}^{0.144}$ & $0.718_{-0.875}^{0.570}$ & $0.419_{-0.384}^{0.514}$ & $0.285_{-0.347}^{0.537}$ & $1.523_{-0.690}^{1.427}$ & 0.32 & 0.28 & 15.7 & 22.7 \\
\hline Year 3 & $0.374_{-0.222}^{0.249}$ & $0.185_{-0.126}^{0.120}$ & $0.733_{-1.031}^{0.527}$ & $0.343_{-0.262}^{0.367}$ & $0.159_{-0.252}^{0.420}$ & $1.004_{-0.647}^{1.554}$ & 0.29 & 0.27 & 18.1 & 26.6 \\
\hline Year 4 & $0.304_{-0.241}^{0.307}$ & $0.214_{-0.154}^{0.148}$ & $0.733_{-0.864}^{0.473}$ & $0.320_{-0.255}^{0.298}$ & $0.100_{-0.328}^{0.983}$ & $0.939_{-0.522}^{1.621}$ & 0.33 & 0.32 & 15.3 & 23.1 \\
\hline Whole sample & $0.636_{-0.183}^{0.179}$ & $0.238_{-0.108}^{0.107}$ & $0.813_{-0.792}^{0.484}$ & $0.548_{-0.366}^{0.437}$ & $0.273_{-0.268}^{0.306}$ & $1.381_{-0.735}^{1.305}$ & 0.35 & 0.29 & 28.3 & 35.7 \\
\hline
\end{tabular}

Notes. Prior for $b, x_{0}$ and $y_{0}$ is a uniform prior with lower and upper limits (e.g., : for $\left.x_{0}: \mathcal{U}(\min (x), \max (x))\right)$ and for $a, k_{1}$ and $k_{2}$ is $\mathcal{U}(0,4)$.

We compare the piecewise two power law function fit and single power law function fit in the peak-to-peak of photometric variability and RV jitter correlation plot. We used Markov-chain Monte Carlo (MCMC) sampling using the library emcee (Foreman-Mackey et al. 2013) to estimate the uncertainties of the parameters in both models.

For the piecewise function we used

$\log 10(y)= \begin{cases}k_{1} \log 10(x)+y_{0}-k_{1} x_{0} & \log (x)<x_{0} \\ k_{2} \log 10(x)+y_{0}-k_{2} x_{0} & \log (x) \geqslant x_{0}\end{cases}$

which here $\left(10^{x_{0}}, 10^{y_{0}}\right)$ is the knee point coordination in the plots. We also fit a single power law function fit as,

$\log 10(y)=a \log 10(x)+b$.

We derived $\mathrm{RMS}_{\text {resi }}$ and BIC value in order to compare the significance of the two models. All best fitted parameters are presented in Table. A.1.

\section{Appendix B: Correlation with other observable}

By having the effective temperature estimated, we investigated the correlation between $T_{\text {eff }}$ and RV-RMS versus peak-to-peak photometric variability. In Fig. B.3, we used $T_{\text {eff }}$ for the color bar. We cannot confirm a significant dependency of the plots on $T_{\text {eff. }}$.

We also inspected if the correlation between RV and photometric jitter has any dependency on the $[\mathrm{Fe} / \mathrm{H}]$, and also the

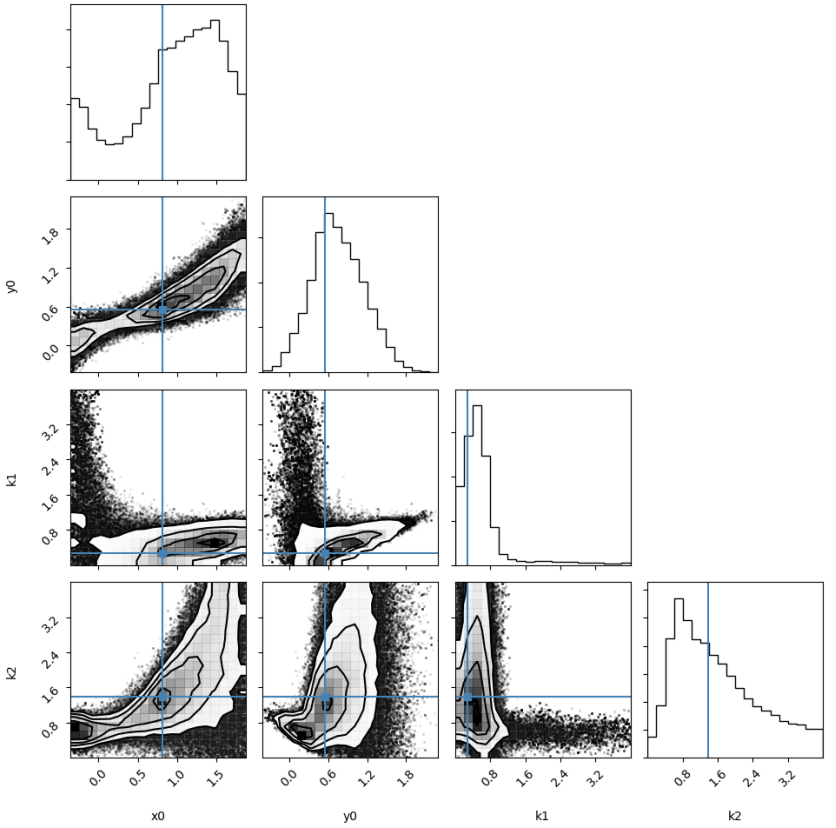

Fig. B.1. Posterior distribution for parameters $x_{0}, y_{0}, k_{1}, k_{2}$ in Eq. (A.1).

number of nights that the star has been observed in RV (see in Fig. B.4). We did not find significant correlation between these parameters. 
S. Hojjatpanah et al.: The correlation between photometric variability and radial velocity jitter

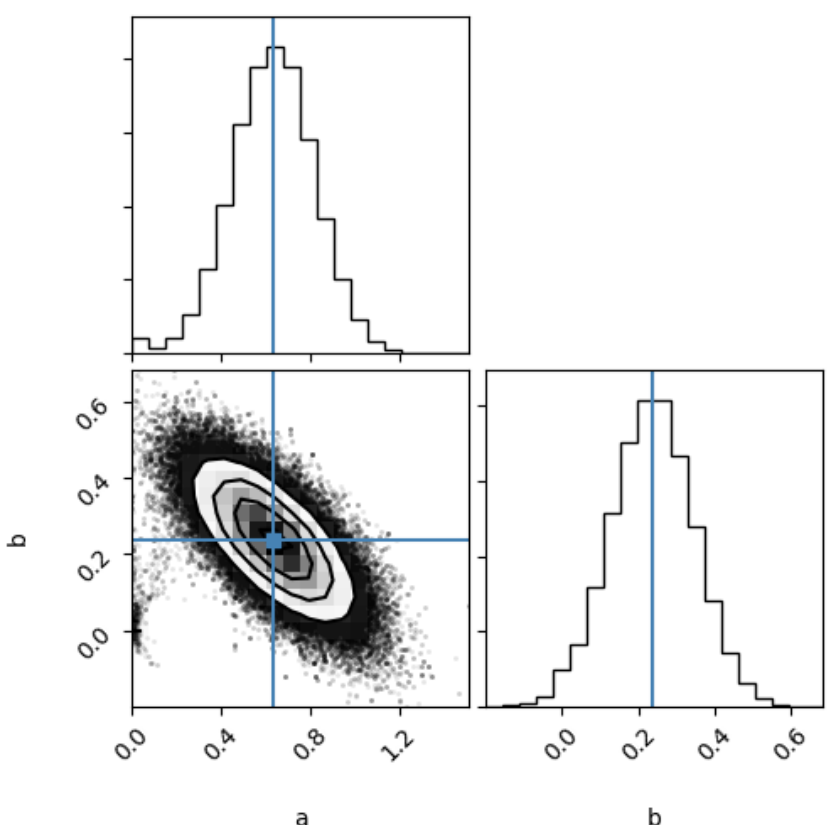

Fig. B.2. Posterior distribution for parameters a, b in Eq. (A.2).

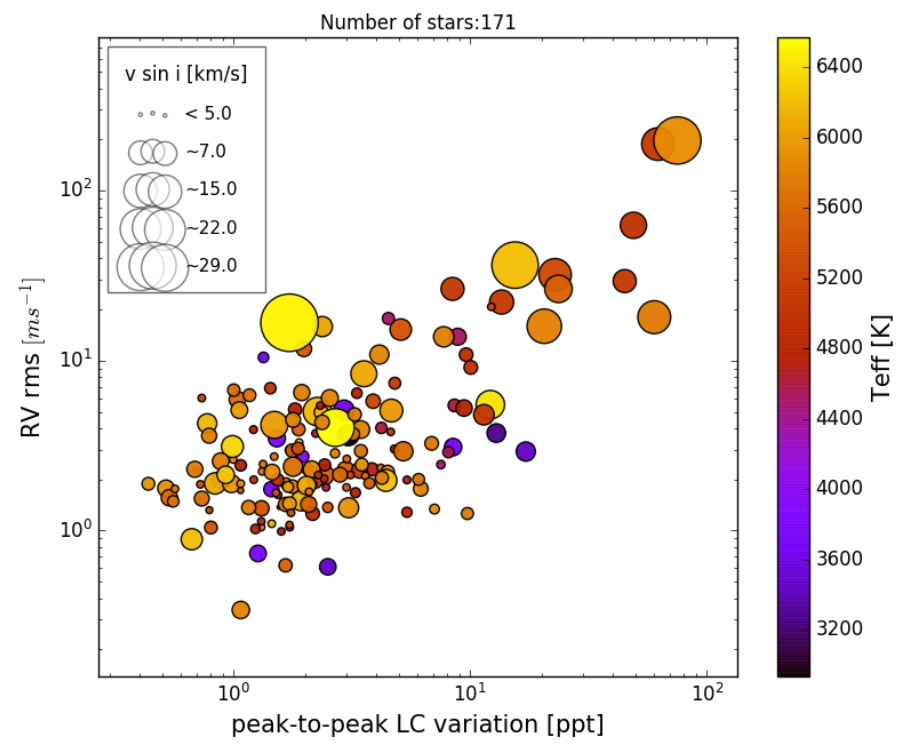

Fig. B.3. Same plots as Fig. 7 with color bar $T_{\text {eff }}$.

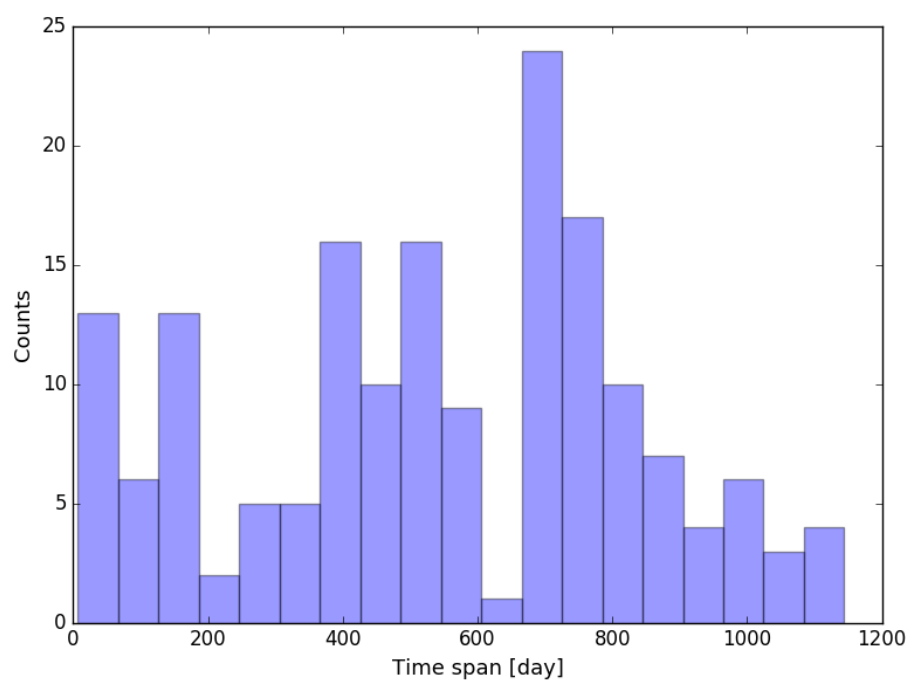

Fig. B.4. Time spans histogram of RV measurements in the sample. 
Appendix C: Table: stellar parameters

Table C.1. Derived parameters for the stars.

\begin{tabular}{|c|c|c|c|c|c|c|c|c|c|c|}
\hline Index & Star & $\begin{array}{l}T_{\text {eff }} \\
{[\mathrm{K}]}\end{array}$ & $\begin{array}{c}\mathrm{RV}^{\mathrm{RMMS}}{ }^{(a)} \\
{\left[\mathrm{m} \mathrm{s}^{-1}\right]}\end{array}$ & $\begin{array}{c}\text { pp Light curves } \\
\text { [ppt] }\end{array}$ & $\begin{array}{l}v \sin i^{(b)} \\
{\left[\mathrm{km} \mathrm{s}^{-1}\right]}\end{array}$ & Mean $\left(\log \left(R_{\mathrm{HK}}^{\prime}\right)\right)^{(c)}$ & $\begin{array}{c}\log g \\
{\left[\mathrm{~cm} \mathrm{~s}^{-2}\right]}\end{array}$ & {$[\mathrm{Fe} / \mathrm{H}]$} & $\begin{array}{c}P_{\text {rot }} \\
\text { [day] }\end{array}$ & Sample ${ }^{(d)}$ \\
\hline 1 & GJ 1061 & $2934.0 \pm 90$ & 3.65 & 3.055 & 4.0 & -4.74 & - & $-0.08 \pm 0.05$ & - & 1 \\
\hline 2 & GJ 180 & $3662.0 \pm 90$ & 10.456 & 1.339 & 1.0 & -5.4 & - & $-0.24 \pm 0.05$ & - & 4 \\
\hline 3 & GJ 3135 & $3649.0 \pm 90$ & 5.105 & 2.931 & 3.7 & -4.95 & - & $-0.34 \pm 0.05$ & - & $2,3,4$ \\
\hline 4 & GJ 3440 & $3693.0 \pm 90$ & 3.101 & 8.47 & 2.7 & -4.89 & - & - & $19.5 \pm 1.2$ & 3 \\
\hline 5 & GJ 588 & $3459.0 \pm 90$ & 0.612 & 2.503 & 2.4 & -5.14 & - & $0.06 \pm 0.05$ & - & 3 \\
\hline 6 & Gl 213 & $3266.0 \pm 90$ & 3.748 & 12.893 & 3.1 & -4.94 & - & $-0.16 \pm 0.05$ & - & 3,4 \\
\hline 7 & Gl 229 & $3785.0 \pm 90$ & 3.488 & 1.525 & 2.6 & -4.95 & - & $-0.02 \pm 0.05$ & - & 3,4 \\
\hline 8 & Gl 693 & $3457.0 \pm 90$ & 2.919 & 17.185 & 3.3 & -4.86 & - & $-0.275 \pm 0.05$ & - & 3,4 \\
\hline 9 & Gl 87 & $3726.0 \pm 90$ & 1.752 & 1.445 & 2.0 & -5.4 & - & $-0.35 \pm 0.05$ & - & 3,4 \\
\hline 10 & HD 149189 & $3726.0 \pm 90$ & 0.733 & 1.269 & 2.4 & -5.09 & - & $0.19 \pm 0.05$ & - & 3 \\
\hline 11 & HD 000105 & $6221.0 \pm 94$ & 36.513 & 15.471 & 19.1 & -4.36 & $4.71 \pm 0.09$ & $-0.13 \pm 0.068$ & $5.3 \pm 1.9$ & $\mathrm{w}$ \\
\hline 12 & HD 007661 & $5410.0 \pm 30$ & 15.244 & 5.088 & 4.1 & -4.4 & $4.38 \pm 0.05$ & $-0.01 \pm 0.022$ & $6.3 \pm 1.1$ & $\mathrm{w}$ \\
\hline 13 & HD 017925 & $5086.0 \pm 41$ & 29.416 & 44.961 & 4.7 & -4.32 & $4.29 \pm 0.09$ & $0.075 \pm 0.023$ & $7.2 \pm 2.6$ & 2 \\
\hline 14 & HD 018599 & $5077.0 \pm 38$ & 26.463 & 8.423 & 4.7 & -4.42 & $4.34 \pm 0.08$ & $-0.01 \pm 0.022$ & $5.4 \pm 0.7$ & 3 \\
\hline 15 & HD 021175 & $5263.0 \pm 39$ & 5.773 & 3.889 & 1.8 & -4.78 & $4.33 \pm 0.07$ & $0.12 \pm 0.026$ & $27.1 \pm 2.3$ & $\mathrm{w}$ \\
\hline 16 & HD 024916 & $4644.0 \pm 97$ & 5.454 & 8.58 & 1.4 & -4.61 & $4.14 \pm 0.36$ & $-0.097 \pm 0.042$ & $16.0 \pm 3.9$ & $\mathrm{w}$ \\
\hline 17 & HD 037572 & $5314.0 \pm 36$ & 32.029 & 22.83 & 9.3 & -4.59 & $4.4 \pm 0.07$ & $-0.344 \pm 0.023$ & $5.6 \pm 3.0$ & $\mathrm{w}$ \\
\hline 18 & HD 092945 & $5091.0 \pm 38$ & 22.13 & 13.576 & 5.1 & -4.35 & $4.39 \pm 0.08$ & $-0.04 \pm 0.022$ & $6.2 \pm 11.1$ & 3 \\
\hline 19 & HD 102438 & $5547.0 \pm 12$ & 2.277 & 2.98 & 2.5 & -4.97 & $4.42 \pm 0.03$ & $-0.29 \pm 0.01$ & - & 3 \\
\hline 20 & HD 10360 & $5026.0 \pm 31$ & 3.808 & 4.619 & 0.5 & -4.8 & $4.37 \pm 0.07$ & $-0.205 \pm 0.017$ & - & 3 \\
\hline 21 & HD 103743 & $5763.0 \pm 33$ & 18.031 & 59.935 & 9.5 & -4.37 & $4.55 \pm 0.04$ & $-0.02 \pm 0.025$ & - & 3 \\
\hline 22 & HD 103891 & $6053.0 \pm 20$ & 15.874 & 2.373 & 3.6 & -4.9 & $3.99 \pm 0.03$ & $-0.2 \pm 0.016$ & - & 24 \\
\hline 23 & HD 107094 & $5522.0 \pm 27$ & 1.742 & 6.351 & 0.5 & -4.85 & $4.43 \pm 0.03$ & $-0.51 \pm 0.021$ & - & w \\
\hline 24 & HD 109200 & $5040.0 \pm 30$ & 1.373 & 1.562 & 0.2 & -4.98 & $4.27 \pm 0.07$ & $-0.285 \pm 0.018$ & $17.4 \pm 0.7$ & 3,4 \\
\hline 25 & HD 111777 & $5622.0 \pm 22$ & 1.446 & 1.649 & 0.5 & -4.93 & $4.37 \pm 0.04$ & $-0.7 \pm 0.018$ & - & 3,4 \\
\hline 26 & HD 114853 & $5668.0 \pm 13$ & 2.262 & 0.698 & 0.4 & -4.96 & $4.36 \pm 0.02$ & $-0.23 \pm 0.01$ & - & $2,3,4$ \\
\hline 27 & HD 11507 & $3991.0 \pm 90$ & 2.725 & 1.965 & 1.3 & -4.8 & - & $-0.21 \pm 0.05$ & - & $\mathrm{w}$ \\
\hline 28 & HD 11608 & $4864.0 \pm 106$ & 1.259 & 2.161 & 1.6 & -4.91 & $4.08 \pm 0.23$ & $0.22 \pm 0.057$ & $8.8 \pm 2.1$ & $1,2,3$ \\
\hline 29 & HD 118466 & $4967.0 \pm 72$ & 10.849 & 9.613 & 1.6 & -5.06 & $4.1 \pm 0.19$ & $0.2 \pm 0.038$ & - & 4 \\
\hline 30 & HD 118563 & $5502.0 \pm 22$ & 11.73 & 1.984 & 2.1 & -4.79 & $4.39 \pm 0.05$ & $-0.04 \pm 0.017$ & - & 4 \\
\hline 31 & HD 121004 & $5672.0 \pm 27$ & 2.639 & 3.012 & 0.5 & -4.93 & $4.39 \pm 0.03$ & $-0.71 \pm 0.021$ & - & 4 \\
\hline 32 & HD 123651 & $5877.0 \pm 27$ & 1.048 & 1.308 & 0.5 & -4.86 & $4.4 \pm 0.03$ & $-0.48 \pm 0.022$ & $14.7 \pm 4.9$ & $\mathrm{w}$ \\
\hline 33 & HD 125072 & $4731.0 \pm 126$ & 2.293 & 3.845 & 1.5 & -4.97 & $3.95 \pm 0.3$ & $0.23 \pm 0.058$ & $13.6 \pm 2.8$ & 3,4 \\
\hline 34 & HD 126803 & $5455.0 \pm 19$ & 1.794 & 3.005 & 0.5 & -4.93 & $4.42 \pm 0.04$ & $-0.63 \pm 0.016$ & - & $2,3,4$ \\
\hline 35 & HD 128571 & $6156.0 \pm 32$ & 1.098 & 1.451 & 0.5 & -4.93 & $4.26 \pm 0.04$ & $-0.37 \pm 0.021$ & $36.3 \pm 6.5$ & 4 \\
\hline 36 & HD 129642 & $4851.0 \pm 46$ & 1.282 & 5.408 & 0.9 & -5.08 & $4.18 \pm 0.14$ & $-0.09 \pm 0.026$ & - & $2,3,4$ \\
\hline 37 & HD 133633 & $5557.0 \pm 24$ & 2.128 & 2.409 & 3.6 & -4.95 & $4.38 \pm 0.03$ & $-0.45 \pm 0.019$ & - & 3,4 \\
\hline 38 & HD 134606 & $5619.0 \pm 30$ & 5.906 & 1.039 & 2.4 & -5.11 & $4.28 \pm 0.07$ & $0.27 \pm 0.023$ & $11.6 \pm 2.0$ & $\mathrm{w}$ \\
\hline 39 & HD 137676 & $5249.0 \pm 16$ & 1.961 & 2.212 & 2.0 & -5.1 & $3.88 \pm 0.04$ & $-0.53 \pm 0.013$ & - & 3,4 \\
\hline 40 & HD 13808 & $5034.0 \pm 36$ & 6.884 & 1.432 & 1.1 & -4.87 & $4.34 \pm 0.08$ & $-0.2 \pm 0.02$ & - & 3 \\
\hline 41 & HD 1388 & $5920.0 \pm 13$ & 1.772 & 0.519 & 2.4 & -4.99 & $4.33 \pm 0.02$ & $-0.01 \pm 0.011$ & $18.3 \pm 2.6$ & $2,3,4$ \\
\hline 42 & HD 141624 & $5887.0 \pm 18$ & 2.14 & 2.27 & 0.5 & -4.94 & $4.4 \pm 0.04$ & $-0.38 \pm 0.014$ & - & 3,4 \\
\hline 43 & HD 142709 & $4573.0 \pm 132$ & 1.795 & 2.463 & 0.5 & -4.98 & $4.44 \pm 0.34$ & $-0.32 \pm 0.031$ & - & 4 \\
\hline 44 & HD 14412 & $5344.0 \pm 21$ & 1.871 & 0.725 & 0.5 & -4.85 & $4.42 \pm 0.05$ & $-0.47 \pm 0.017$ & $13.0 \pm 0.3$ & $1,2,3$ \\
\hline 45 & HD 144628 & $4979.0 \pm 42$ & 2.021 & 2.416 & 0.5 & -4.89 & $4.34 \pm 0.08$ & $-0.36 \pm 0.024$ & - & 3,4 \\
\hline 46 & HD 14745 & $6182.0 \pm 27$ & 4.274 & 0.774 & 3.3 & -5.0 & $4.42 \pm 0.02$ & $-0.14 \pm 0.019$ & - & 2,3 \\
\hline 47 & HD 150139 & $5904.0 \pm 26$ & 2.62 & 0.999 & 0.5 & -4.91 & $4.16 \pm 0.03$ & $-0.51 \pm 0.02$ & - & 4 \\
\hline 48 & HD 150474 & $5423.0 \pm 18$ & 1.352 & 1.313 & 2.1 & -5.15 & $3.93 \pm 0.03$ & $0.01 \pm 0.015$ & - & 1,2 \\
\hline 49 & HD 154577 & $4868.0 \pm 36$ & 1.623 & 1.543 & 0.5 & -4.87 & $4.44 \pm 0.09$ & $-0.65 \pm 0.018$ & - & 3,4 \\
\hline 50 & HD 156098 & $6418.0 \pm 37$ & 5.506 & 12.165 & 7.2 & -4.82 & $3.86 \pm 0.05$ & $0.12 \pm 0.026$ & - & 4 \\
\hline 51 & HD 1581 & $5946.0 \pm 17$ & 1.885 & 0.437 & 1.4 & -4.95 & $4.44 \pm 0.03$ & $-0.2 \pm 0.013$ & - & $2,3,4$ \\
\hline 52 & HD 16280 & $4625.0 \pm 168$ & 4.02 & 4.216 & 1.1 & -4.82 & $4.22 \pm 0.41$ & $-0.19 \pm 0.103$ & $14.8 \pm 3.9$ & 13 \\
\hline 53 & HD 16548 & $5685.0 \pm 15$ & 2.377 & 1.785 & 3.3 & -5.16 & $3.95 \pm 0.04$ & $0.15 \pm 0.012$ & - & $\mathrm{w}$ \\
\hline 54 & HD 16905 & $4760.0 \pm 104$ & 5.159 & 1.821 & 1.5 & -4.77 & $4.14 \pm 0.27$ & $0.15 \pm 0.06$ & $23.8 \pm 0.2$ & 2 \\
\hline 55 & HD 172568 & $5690.0 \pm 18$ & 1.865 & 2.176 & 0.5 & -4.93 & $4.41 \pm 0.03$ & $-0.37 \pm 0.014$ & - & 3,4 \\
\hline 56 & HD 17970 & $5005.0 \pm 30$ & 1.994 & 1.218 & 0.5 & -5.0 & $4.34 \pm 0.08$ & $-0.45 \pm 0.019$ & - & 2,3 \\
\hline 57 & HD 181327 & $6502.0 \pm 90$ & 16.722 & 1.724 & 29.1 & -4.57 & - & $0.055 \pm 0.05$ & $2.3 \pm 4.6$ & 4 \\
\hline 58 & HD 183414 & $5816.0 \pm 33$ & 15.956 & 20.565 & 10.4 & -4.34 & $4.51 \pm 0.04$ & $-0.165 \pm 0.026$ & $5.6 \pm 1.0$ & 3 \\
\hline 59 & HD 183783 & $4796.0 \pm 77$ & 3.93 & 1.215 & 0.5 & -4.88 & $4.14 \pm 0.47$ & $-0.21 \pm 0.031$ & $5.4 \pm 2.2$ & 4 \\
\hline 60 & HD 185283 & $4754.0 \pm 65$ & 1.671 & 3.277 & 0.9 & -5.01 & $4.24 \pm 0.16$ & $-0.06 \pm 0.03$ & $28.9 \pm 0.6$ & 3,4 \\
\hline 61 & HD 187456 & $4715.0 \pm 90$ & 2.42 & 1.073 & 1.2 & -4.93 & $4.09 \pm 0.24$ & $0.02 \pm 0.054$ & $12.1 \pm 1.6$ & 4 \\
\hline 62 & HD 189567 & $5710.0 \pm 12$ & 2.726 & 1.486 & 0.5 & -4.93 & $4.37 \pm 0.02$ & $-0.24 \pm 0.01$ & - & $\mathrm{w}$ \\
\hline 63 & HD 190248 & $5653.0 \pm 77$ & 1.561 & 0.536 & 2.4 & -5.12 & $4.6 \pm 0.13$ & $0.33 \pm 0.056$ & $21.4 \pm 9.3$ & 3,4 \\
\hline 64 & HD 19230 & $5118.0 \pm 31$ & 7.372 & 4.805 & 1.2 & -4.79 & $4.37 \pm 0.06$ & $-0.57 \pm 0.021$ & $13.5 \pm 4.1$ & 1 \\
\hline
\end{tabular}

Notes. ${ }^{(a)}$ Using the whole sample, ${ }^{(b)}$ mean $v \sin i$ error: $1.0\left[\mathrm{~km} \mathrm{~s}^{-1}\right],{ }^{(c)}$ mean $\log \left(R_{\mathrm{HK}}^{\prime}\right)$ error: $0.01,{ }^{(d)}$ subsample classifications, w: only considered in whole sample. 
Table C.1. continued.

\begin{tabular}{|c|c|c|c|c|c|c|c|c|c|c|}
\hline Index & Star & $\begin{array}{l}T_{\text {eff }} \\
{[\mathrm{K}]}\end{array}$ & $\begin{array}{c}\mathrm{RV}^{\mathrm{RMS}}{ }^{(a)} \\
{\left[\mathrm{m} \mathrm{s}^{-1}\right]}\end{array}$ & $\begin{array}{c}\text { pp Light curves } \\
\text { [ppt] }\end{array}$ & $\begin{array}{l}v \sin i^{(b)} \\
{\left[\mathrm{km} \mathrm{s}^{-1}\right]}\end{array}$ & $\operatorname{Mean}\left(\log \left(R_{\mathrm{HK}}^{\prime}\right)\right)^{(c)}$ & $\begin{array}{c}\log g \\
{\left[\mathrm{~cm} \mathrm{~s}^{-2}\right]}\end{array}$ & {$[\mathrm{Fe} / \mathrm{H}]$} & $\begin{array}{c}P_{\text {rot }} \\
\text { [day] }\end{array}$ & Sample ${ }^{(d)}$ \\
\hline 65 & HD 19641 & $5811.0 \pm 12$ & 1.426 & 1.659 & 1.8 & -5.01 & $4.38 \pm 0.03$ & $-0.01 \pm 0.01$ & - & 2 \\
\hline 66 & HD 197481 & $5129.0 \pm 262$ & 188.286 & 62.137 & 9.4 & -4.04 & $4.36 \pm 0.5$ & $0.1 \pm 0.172$ & $6.0 \pm 1.1$ & 1 \\
\hline 67 & HD 199288 & $5756.0 \pm 17$ & 1.76 & 0.566 & 0.5 & -4.92 & $4.47 \pm 0.03$ & $-0.63 \pm 0.014$ & $7.5 \pm 1.3$ & $1,2,3,4$ \\
\hline 68 & HD 199604 & $5785.0 \pm 34$ & 2.338 & 4.192 & 0.5 & -4.94 & $4.27 \pm 0.03$ & $-0.62 \pm 0.027$ & - & $1,2,3$ \\
\hline 69 & HD 199847 & $5722.0 \pm 27$ & 2.23 & 1.321 & 0.5 & -5.02 & $4.14 \pm 0.03$ & $-0.54 \pm 0.021$ & - & 3,4 \\
\hline 70 & HD 199981 & $4559.0 \pm 149$ & 2.893 & 8.093 & 1.1 & -4.68 & $4.3 \pm 0.55$ & $-0.16 \pm 0.05$ & $12.6 \pm 2.2$ & 3 \\
\hline 71 & HD 200133 & $6142.0 \pm 25$ & 1.498 & 1.935 & 3.7 & -5.05 & $4.38 \pm 0.04$ & $0.3 \pm 0.018$ & - & 3 \\
\hline 72 & HD 202917 & $5902.0 \pm 109$ & 197.725 & 75.061 & 19.7 & -4.09 & $5.0 \pm 0.11$ & $-0.095 \pm 0.076$ & $3.6 \pm 1.0$ & $\mathrm{w}$ \\
\hline 73 & HD 203850 & $4793.0 \pm 52$ & 1.046 & 1.729 & 0.5 & -4.79 & $4.43 \pm 0.12$ & $-0.68 \pm 0.021$ & - & 3 \\
\hline 74 & HD 20407 & $5835.0 \pm 16$ & 2.137 & 0.881 & 0.5 & -4.9 & $4.46 \pm 0.02$ & $-0.44 \pm 0.013$ & - & 2,3 \\
\hline 75 & HD 205536 & $5426.0 \pm 20$ & 1.042 & 0.803 & 1.4 & -5.03 & $4.31 \pm 0.04$ & $-0.05 \pm 0.015$ & $3.2 \pm 0.9$ & 4 \\
\hline 76 & HD 206683 & $5945.0 \pm 21$ & 1.362 & 3.065 & 3.5 & -5.05 & $4.35 \pm 0.06$ & $0.24 \pm 0.017$ & - & 3 \\
\hline 77 & HD 206998 & $5775.0 \pm 28$ & 2.133 & 2.099 & 0.5 & -5.01 & $4.14 \pm 0.03$ & $-0.72 \pm 0.021$ & - & $1,2,3$ \\
\hline 78 & HD 207129 & $5919.0 \pm 13$ & 1.761 & 6.185 & 1.9 & -4.91 & $4.43 \pm 0.02$ & $-0.02 \pm 0.01$ & - & 3 \\
\hline 79 & HD 207869 & $5480.0 \pm 18$ & 1.668 & 1.511 & 0.5 & -4.96 & $4.41 \pm 0.03$ & $-0.45 \pm 0.014$ & - & 24 \\
\hline 80 & HD 20807 & $5832.0 \pm 15$ & 1.896 & 1.666 & 1.1 & -4.9 & $4.49 \pm 0.03$ & $-0.23 \pm 0.012$ & _ & 2,3 \\
\hline 81 & HD 209100 & $4776.0 \pm 154$ & 2.287 & 2.061 & 1.2 & -4.77 & $4.59 \pm 0.39$ & $-0.105 \pm 0.057$ & - & 3 \\
\hline 82 & HD 210918 & $5743.0 \pm 10$ & 1.487 & 0.557 & 1.2 & -5.02 & $4.3 \pm 0.02$ & $-0.1 \pm 0.009$ & - & $1,2,3,4$ \\
\hline 83 & HD 211415 & $5839.0 \pm 14$ & 2.209 & 4.479 & 1.7 & -4.97 & $4.42 \pm 0.03$ & $-0.21 \pm 0.011$ & - & 3,4 \\
\hline 84 & HD 21161 & $5916.0 \pm 13$ & 1.985 & 0.886 & 2.7 & -5.06 & $4.26 \pm 0.03$ & $0.09 \pm 0.01$ & - & 1 \\
\hline 85 & HD 212036 & $5683.0 \pm 19$ & 6.71 & 1.003 & 1.3 & -5.05 & $4.34 \pm 0.03$ & $-0.01 \pm 0.014$ & $2.5 \pm 1.4$ & 2 \\
\hline 86 & HD 21209A & $4682.0 \pm 46$ & 1.987 & 5.407 & 0.5 & -4.84 & $4.5 \pm 0.14$ & $-0.41 \pm 0.064$ & $38.8 \pm 6.9$ & 3 \\
\hline 87 & HD 213042 & $4709.0 \pm 125$ & 2.434 & 2.428 & 1.3 & -4.88 & $4.15 \pm 0.4$ & $0.165 \pm 0.057$ & - & 3 \\
\hline 88 & HD 213628 & $5553.0 \pm 22$ & 0.624 & 1.66 & 1.5 & -5.0 & $4.38 \pm 0.04$ & $0.01 \pm 0.017$ & - & 4 \\
\hline 89 & HD 215456 & $5797.0 \pm 11$ & 3.617 & 0.789 & 2.0 & -5.09 & $4.07 \pm 0.02$ & $-0.09 \pm 0.009$ & - & 2,3 \\
\hline 90 & HD 215906 & $6219.0 \pm 29$ & 0.889 & 0.666 & 4.0 & -4.91 & $4.37 \pm 0.03$ & $-0.28 \pm 0.02$ & $2.3 \pm 0.6$ & 3 \\
\hline 91 & HD 216054 & $5380.0 \pm 20$ & 1.081 & 1.725 & 0.4 & -5.01 & $4.37 \pm 0.04$ & $-0.12 \pm 0.015$ & - & 1,2 \\
\hline 92 & HD 218860 & $5557.0 \pm 29$ & 26.463 & 23.599 & 6.7 & -4.31 & $4.43 \pm 0.05$ & $-0.015 \pm 0.022$ & $5.0 \pm 2.7$ & 4 \\
\hline 93 & HD 220339 & $4918.0 \pm 40$ & 3.02 & 4.789 & 0.6 & -4.84 & $4.31 \pm 0.08$ & $-0.31 \pm 0.02$ & - & 3 \\
\hline 94 & HD 220507 & $5672.0 \pm 13$ & 1.543 & 0.735 & 2.0 & -5.07 & $4.22 \pm 0.05$ & $0.01 \pm 0.011$ & _- & $1,2,3$ \\
\hline 95 & HD 221575 & $5065.0 \pm 41$ & 4.806 & 11.43 & 3.7 & -4.49 & $4.4 \pm 0.09$ & $0.01 \pm 0.022$ & $11.0 \pm 3.9$ & w \\
\hline 96 & HD 221638 & $6349.0 \pm 33$ & 3.124 & 0.99 & 4.2 & -4.91 & $4.45 \pm 0.04$ & $-0.225 \pm 0.023$ & - & 1,2 \\
\hline 97 & HD 222237 & $4688.0 \pm 130$ & 2.985 & 1.716 & 0.5 & -4.91 & $4.17 \pm 0.27$ & $-0.31 \pm 0.188$ & - & 3 \\
\hline 98 & HD 222335 & $5237.0 \pm 32$ & 6.024 & 0.735 & 0.5 & -4.8 & $4.4 \pm 0.07$ & $-0.16 \pm 0.022$ & - & $1,2,3$ \\
\hline 99 & HD 222480 & $5840.0 \pm 15$ & 3.932 & 3.453 & 2.7 & -5.04 & $4.15 \pm 0.04$ & $0.19 \pm 0.012$ & - & $1,2,3$ \\
\hline 100 & HD 222669 & $5896.0 \pm 15$ & 2.294 & 2.139 & 2.5 & -4.9 & $4.45 \pm 0.04$ & $0.05 \pm 0.012$ & - & 2 \\
\hline 101 & HD 223171 & $5797.0 \pm 14$ & 2.559 & 0.883 & 2.5 & -5.04 & $4.09 \pm 0.04$ & $0.115 \pm 0.011$ & $13.8 \pm 5.9$ & $1,2,3$ \\
\hline 102 & HD 223681 & $4995.0 \pm 55$ & 62.608 & 48.955 & 6.1 & -4.05 & $4.39 \pm 0.11$ & $-0.555 \pm 0.025$ & $5.6 \pm 1.0$ & 2 \\
\hline 103 & HD 224228 & $4771.0 \pm 59$ & 5.262 & 9.418 & 2.4 & -4.44 & $4.19 \pm 0.15$ & $0.01 \pm 0.028$ & $27.2 \pm 3.9$ & $\mathrm{w}$ \\
\hline 104 & HD 224230 & $4786.0 \pm 79$ & 6.436 & 3.322 & 1.0 & -4.99 & $4.25 \pm 0.19$ & $-0.1 \pm 0.037$ & - & 2 \\
\hline 105 & HD 224619 & $5454.0 \pm 19$ & 1.369 & 2.502 & 0.9 & -4.97 & $4.4 \pm 0.04$ & $-0.2 \pm 0.015$ & $15.8 \pm 3.9$ & 3 \\
\hline 106 & HD 23249 & $5120.0 \pm 128$ & 2.264 & 3.408 & 2.2 & -5.21 & $4.3 \pm 0.24$ & $0.09 \pm 0.074$ & - & 2,3 \\
\hline 107 & HD 24062 & $5990.0 \pm 19$ & 5.086 & 4.646 & 4.6 & -5.06 & $4.22 \pm 0.03$ & $0.23 \pm 0.015$ & - & 3 \\
\hline 108 & HD 25587 & $6207.0 \pm 29$ & 5.015 & 2.262 & 7.2 & -5.0 & $4.26 \pm 0.03$ & $-0.12 \pm 0.022$ & $23.1 \pm 4.1$ & 2 \\
\hline 109 & HD 28471 & $5730.0 \pm 13$ & 1.814 & 1.775 & 1.6 & -5.0 & $4.32 \pm 0.02$ & $-0.05 \pm 0.011$ & $18.7 \pm 7.2$ & 2 \\
\hline 110 & HD 30306 & $5552.0 \pm 25$ & 1.427 & 2.078 & 2.2 & -5.09 & $4.26 \pm 0.05$ & $0.18 \pm 0.019$ & $44.8 \pm 2.3$ & 2 \\
\hline 111 & HD 3074A & $6100.0 \pm 17$ & 1.895 & 0.837 & 4.0 & -5.03 & $4.39 \pm 0.04$ & $-0.01 \pm 0.013$ & $12.2 \pm 0.7$ & 2,3 \\
\hline 112 & HD 30876 & $4985.0 \pm 47$ & 9.128 & 10.06 & 1.7 & -4.52 & $4.36 \pm 0.1$ & $-0.085 \pm 0.024$ & $9.6 \pm 0.8$ & 2 \\
\hline 113 & HD 31822 & $6016.0 \pm 19$ & 5.119 & 1.059 & 2.5 & -4.86 & $4.5 \pm 0.03$ & $-0.19 \pm 0.014$ & $3.6 \pm 0.6$ & 3,4 \\
\hline 114 & HD 32564 & $5518.0 \pm 16$ & 3.944 & 1.884 & 1.5 & -5.02 & $4.36 \pm 0.03$ & $0.08 \pm 0.012$ & - & 3 \\
\hline 115 & HD 35854 & $4788.0 \pm 61$ & 1.023 & 1.238 & 0.8 & -4.82 & $4.2 \pm 0.17$ & $-0.13 \pm 0.03$ & - & 3,4 \\
\hline 116 & HD 36003 & $4558.0 \pm 278$ & 3.732 & 2.226 & 0.6 & -4.89 & $4.13 \pm 0.76$ & $-0.13 \pm 0.038$ & $2.4 \pm 0.7$ & 3,4 \\
\hline 117 & HD 36379 & $6026.0 \pm 15$ & 1.441 & 1.726 & 1.6 & -5.0 & $4.26 \pm 0.03$ & $-0.17 \pm 0.011$ & $2.8 \pm 1.0$ & 2,3 \\
\hline 118 & HD 37990 & $6201.0 \pm 24$ & 4.961 & 2.348 & 1.7 & -4.75 & $4.48 \pm 0.04$ & $-0.03 \pm 0.017$ & $12.3 \pm 2.2$ & 3 \\
\hline 119 & HD 3823 & $6008.0 \pm 17$ & 1.807 & 1.701 & 1.0 & -5.01 & $4.28 \pm 0.04$ & $-0.28 \pm 0.013$ & - & 2,3 \\
\hline 120 & HD 38459 & $5320.0 \pm 38$ & 20.766 & 12.281 & 0.5 & -4.51 & $4.28 \pm 0.1$ & $0.11 \pm 0.026$ & $12.0 \pm 2.2$ & 2 \\
\hline 121 & HD 38858 & $5705.0 \pm 13$ & 2.098 & 3.607 & 0.5 & -4.94 & $4.46 \pm 0.03$ & $-0.23 \pm 0.01$ & - & 3 \\
\hline 122 & HD 39194 & $5188.0 \pm 17$ & 2.935 & 1.768 & 0.5 & -4.96 & $4.47 \pm 0.04$ & $-0.61 \pm 0.012$ & $20.4 \pm 6.5$ & 3 \\
\hline 123 & HD 3964 & $5723.0 \pm 15$ & 3.26 & 6.874 & 1.7 & -4.9 & $4.43 \pm 0.02$ & $0.05 \pm 0.012$ & - & 1,2 \\
\hline 124 & HD 40397 & $5504.0 \pm 18$ & 1.899 & 3.75 & 1.1 & -5.02 & $4.35 \pm 0.04$ & $-0.13 \pm 0.014$ & $2.7 \pm 0.5$ & 3,4 \\
\hline 125 & HD 40865 & $5678.0 \pm 18$ & 2.46 & 1.333 & 0.5 & -4.94 & $4.39 \pm 0.03$ & $-0.38 \pm 0.015$ & - & 2,3 \\
\hline 126 & HD 42936 & $5075.0 \pm 60$ & 2.123 & 2.281 & 0.5 & -5.1 & $4.18 \pm 0.16$ & $0.18 \pm 0.039$ & - & 2 \\
\hline 127 & HD 52449 & $6342.0 \pm 23$ & 3.295 & 1.895 & 0.5 & -4.74 & $4.49 \pm 0.03$ & $0.09 \pm 0.017$ & $27.7 \pm 5.7$ & 1,2 \\
\hline 128 & HD 53705 & $5765.0 \pm 29$ & 1.26 & 9.736 & 1.3 & -5.01 & $4.35 \pm 0.05$ & $-0.227 \pm 0.023$ & - & 4 \\
\hline 129 & HD 56380 & $5310.0 \pm 25$ & 2.124 & 3.119 & 0.5 & -5.0 & $4.3 \pm 0.04$ & $-0.42 \pm 0.018$ & - & 2,3 \\
\hline 130 & HD 6107 & $5797.0 \pm 13$ & 2.296 & 0.687 & 2.3 & -5.06 & $4.04 \pm 0.02$ & $-0.055 \pm 0.011$ & $13.6 \pm 5.8$ & $1,2,3$ \\
\hline 131 & HD 65277 & $4646.0 \pm 69$ & 1.132 & 1.311 & 0.5 & -5.04 & $4.33 \pm 0.17$ & $-0.31 \pm 0.03$ & $25.7 \pm 4.6$ & 3 \\
\hline
\end{tabular}


Table C.1. continued.

\begin{tabular}{|c|c|c|c|c|c|c|c|c|c|c|}
\hline Index & Star & $\begin{array}{l}T_{\text {eff }} \\
{[\mathrm{K}]}\end{array}$ & $\begin{array}{c}\mathrm{RV}-\mathrm{RMS}^{(a)} \\
{\left[\mathrm{m} \mathrm{s}^{-1}\right]}\end{array}$ & $\begin{array}{l}\text { pp Light curves } \\
\text { [ppt] }\end{array}$ & $\begin{array}{l}v \sin i^{(b)} \\
{\left[\mathrm{km} \mathrm{s}^{-1}\right]}\end{array}$ & $\operatorname{Mean}\left(\log \left(R_{\mathrm{HK}}^{\prime}\right)\right)^{(c)}$ & $\begin{array}{c}\log g \\
{\left[\mathrm{~cm} \mathrm{~s}^{-2}\right]}\end{array}$ & {$[\mathrm{Fe} / \mathrm{H}]$} & $\begin{array}{c}P_{\text {rot }} \\
\text { [day] }\end{array}$ & Sample ${ }^{(d)}$ \\
\hline 132 & HD 65907A & $5911.0 \pm 80$ & 1.338 & 7.067 & 0.8 & -4.94 & $4.57 \pm 0.09$ & $-0.31 \pm 0.061$ & $13.8 \pm 2.2$ & 3 \\
\hline 133 & HD 67200 & $6090.0 \pm 18$ & 1.843 & 2.036 & 3.0 & -5.06 & $4.36 \pm 0.04$ & $0.32 \pm 0.014$ & $34.1 \pm 6.1$ & 2 \\
\hline 134 & HD 69611 & $5776.0 \pm 22$ & 1.69 & 2.087 & 0.5 & -4.98 & $4.3 \pm 0.03$ & $-0.58 \pm 0.017$ & - & 2,3 \\
\hline 135 & HD 71835 & $5475.0 \pm 24$ & 2.957 & 1.768 & 1.5 & -4.96 & $4.41 \pm 0.04$ & $-0.04 \pm 0.017$ & - & 2,3 \\
\hline 136 & HD 72673 & $5201.0 \pm 21$ & 1.319 & 0.791 & 0.4 & -4.94 & $4.42 \pm 0.04$ & $-0.38 \pm 0.015$ & - & $2,3,4$ \\
\hline 137 & HD 73524 & $5979.0 \pm 17$ & 1.886 & 0.981 & 2.8 & -5.03 & $4.36 \pm 0.03$ & $0.15 \pm 0.013$ & - & $2,3,4$ \\
\hline 138 & HD 74698 & $5797.0 \pm 13$ & 6.49 & 1.943 & 2.3 & -5.07 & $4.23 \pm 0.03$ & $0.07 \pm 0.01$ & - & 1,24 \\
\hline 139 & HD 75881 & $6094.0 \pm 28$ & 8.375 & 3.554 & 5.9 & -5.15 & $4.06 \pm 0.04$ & $0.07 \pm 0.022$ & - & w \\
\hline 140 & HD 76849 & $5285.0 \pm 36$ & 1.272 & 1.75 & 0.5 & -5.04 & $4.34 \pm 0.09$ & - & - & 4 \\
\hline 141 & HD 78429 & $5738.0 \pm 12$ & 3.698 & 3.201 & 1.5 & -4.93 & $4.25 \pm 0.03$ & $0.089 \pm 0.01$ & $34.4 \pm 4.2$ & 2,3 \\
\hline 142 & HD 82342 & $4728.0 \pm 607$ & 0.989 & 1.592 & 0.5 & -5.0 & $4.23 \pm 1.21$ & $-0.54 \pm 1.145$ & $30.8 \pm 4.9$ & 3 \\
\hline 143 & HD 82516 & $4998.0 \pm 43$ & 1.989 & 1.536 & 1.3 & -5.01 & $4.24 \pm 0.12$ & $0.01 \pm 0.023$ & $10.6 \pm 1.9$ & $2,3,4$ \\
\hline 144 & HD 85249 & $6567.0 \pm 51$ & 4.04 & 2.693 & 12.6 & -4.92 & $4.63 \pm 0.07$ & $0.18 \pm 0.035$ & - & 3 \\
\hline 145 & HD 85725 & $6000.0 \pm 26$ & 4.187 & 1.488 & 6.5 & -5.19 & $4.0 \pm 0.05$ & $0.15 \pm 0.02$ & - & $\mathrm{w}$ \\
\hline 146 & HD 87838 & $5993.0 \pm 33$ & 1.888 & 1.072 & 0.5 & -4.94 & $4.24 \pm 0.03$ & $-0.4 \pm 0.023$ & $27.4 \pm 4.9$ & $2,3,4$ \\
\hline 147 & HD 88218 & $5845.0 \pm 14$ & 4.48 & 1.785 & 1.9 & -5.05 & $4.09 \pm 0.02$ & $-0.14 \pm 0.012$ & - & 3,4 \\
\hline 148 & HD 8828 & $5386.0 \pm 21$ & 1.722 & 1.072 & 0.3 & -5.01 & $4.4 \pm 0.03$ & $-0.16 \pm 0.016$ & - & 2,3 \\
\hline 149 & HD 89839 & $6245.0 \pm 25$ & 1.973 & 4.409 & 4.2 & -5.01 & $4.4 \pm 0.04$ & $0.04 \pm 0.018$ & - & 2 \\
\hline 150 & HD 92719 & $5794.0 \pm 14$ & 4.34 & 2.368 & 1.9 & -4.85 & $4.43 \pm 0.02$ & $-0.11 \pm 0.011$ & $9.5 \pm 1.7$ & 4 \\
\hline 151 & HD 93489 & $5910.0 \pm 13$ & 2.21 & 1.456 & 2.2 & -5.0 & $4.36 \pm 0.02$ & $-0.02 \pm 0.011$ & - & 2,3 \\
\hline 152 & HD 94151 & $5625.0 \pm 17$ & 2.131 & 2.82 & 1.9 & -4.93 & $4.41 \pm 0.03$ & $0.04 \pm 0.013$ & $10.6 \pm 1.9$ & 3 \\
\hline 153 & HD 94270 & $5983.0 \pm 11$ & 2.443 & 3.483 & 1.5 & -5.0 & $4.37 \pm 0.02$ & $0.02 \pm 0.009$ & $5.9 \pm 2.0$ & 3 \\
\hline 154 & HD 94771 & $5618.0 \pm 16$ & 2.93 & 5.22 & 3.3 & -5.18 & $3.93 \pm 0.04$ & $0.22 \pm 0.013$ & - & 2,4 \\
\hline 155 & HD 95456 & $6292.0 \pm 17$ & 2.13 & 0.927 & 2.7 & -5.02 & $4.39 \pm 0.04$ & $0.16 \pm 0.013$ & - & $2,3,4$ \\
\hline 156 & HD 96700 & $5838.0 \pm 12$ & 2.847 & 2.685 & 0.8 & -4.96 & $4.34 \pm 0.02$ & $-0.18 \pm 0.01$ & $6.0 \pm 1.1$ & 4 \\
\hline 157 & HD 97343 & $5390.0 \pm 26$ & 3.043 & 1.873 & 1.4 & -5.02 & $4.32 \pm 0.04$ & $-0.055 \pm 0.019$ & - & 3,4 \\
\hline 158 & HIP102152 & $5706.0 \pm 13$ & 2.939 & 3.455 & 1.5 & -5.02 & $4.3 \pm 0.03$ & $-0.03 \pm 0.01$ & - & 2,3 \\
\hline 159 & HIP105184 & $5831.0 \pm 13$ & 6.028 & 2.552 & 2.5 & -4.65 & $4.46 \pm 0.02$ & $-0.02 \pm 0.011$ & - & 3 \\
\hline 160 & HIP114328 & $5778.0 \pm 13$ & 2.046 & 4.235 & 1.5 & -5.03 & $4.34 \pm 0.03$ & $-0.017 \pm 0.011$ & - & 4 \\
\hline 161 & HIP114615 & $5784.0 \pm 16$ & 4.824 & 3.251 & 1.6 & -4.78 & $4.4 \pm 0.02$ & $-0.057 \pm 0.013$ & $24.7 \pm 0.4$ & 3,4 \\
\hline 162 & HIP17157 & $4534.0 \pm 150$ & 13.832 & 8.864 & 2.5 & -4.64 & $4.27 \pm 0.48$ & - & $15.0 \pm 2.7$ & 1,2 \\
\hline 163 & HIP21934 & $4507.0 \pm 247$ & 17.715 & 4.513 & 1.3 & -4.93 & $4.07 \pm 0.71$ & $0.03 \pm 0.149$ & $30.3 \pm 0.9$ & $1,2,3,4$ \\
\hline 164 & HIP22263 & $5836.0 \pm 31$ & 10.863 & 4.133 & 3.2 & -4.56 & $4.54 \pm 0.06$ & - & $10.6 \pm 1.9$ & 2 \\
\hline 165 & HIP25670 & $5768.0 \pm 12$ & 1.368 & 1.16 & 1.8 & -4.99 & $4.39 \pm 0.03$ & $0.06 \pm 0.009$ & $10.1 \pm 1.8$ & $2,3,4$ \\
\hline 166 & HIP28066 & $5695.0 \pm 12$ & 2.0 & 6.057 & 1.2 & -5.03 & $4.23 \pm 0.02$ & $-0.147 \pm 0.01$ & - & 2 \\
\hline 167 & HIP36515 & $5801.0 \pm 17$ & 13.82 & 7.731 & 3.7 & -4.42 & $4.45 \pm 0.03$ & $-0.07 \pm 0.013$ & $5.3 \pm 2.2$ & 2 \\
\hline 168 & HIP41317 & $5698.0 \pm 11$ & 6.261 & 1.169 & 1.4 & -5.02 & $4.37 \pm 0.02$ & $-0.081 \pm 0.009$ & - & 3 \\
\hline 169 & HIP54597 & $4679.0 \pm 87$ & 5.441 & 2.325 & 0.5 & -5.04 & $4.23 \pm 0.23$ & $-0.22 \pm 0.04$ & - & $2,3,4$ \\
\hline 170 & HIP68468 & $5848.0 \pm 14$ & 0.341 & 1.074 & 2.7 & -5.06 & $4.32 \pm 0.03$ & $0.07 \pm 0.012$ & - & 3 \\
\hline 171 & HIP79361 & $4490.0 \pm 226$ & 2.446 & 7.522 & 0.6 & -4.87 & $4.11 \pm 0.55$ & $0.52 \pm 0.467$ & - & 3 \\
\hline
\end{tabular}


S. Hojjatpanah et al.: The correlation between photometric variability and radial velocity jitter

Table C.2. Starts in Fig. 14 with the peak-to-peak of light curve variation $<6.5$ ppt and some stellar parameters presents in TIC (Stassun et al. 2018).

\begin{tabular}{|c|c|c|c|c|c|c|}
\hline TIC ID & Full TOI id & $\begin{array}{c}\text { Peak-to-peak light curve } \\
{[\mathrm{ppt}]}\end{array}$ & TESS mag & $\begin{array}{l}T_{\text {eff }} \\
{[\mathrm{K}]}\end{array}$ & $\begin{array}{c}R_{\text {star }} \\
{\left[R_{\text {Sun }}\right]}\end{array}$ & $\begin{array}{c}\log g \\
{\left[\mathrm{~cm} \mathrm{~s}^{-2}\right]}\end{array}$ \\
\hline 9033144 & 367.01 & 6.02 & 9.7 & $5756.9 \pm 195.2$ & $1.3 \pm 4.0$ & $3.9 \pm 0.1$ \\
\hline 9725627 & 239.01 & 5.89 & 11.0 & $6362.5 \pm 83.0$ & $0.9 \pm 1.2$ & $4.6 \pm 0.2$ \\
\hline 9727392 & 236.01 & 6.5 & 11.2 & $6421.5 \pm 83.0$ & $1.5 \pm 5.1$ & $4.2 \pm 0.2$ \\
\hline 13021029 & 439.01 & 5.4 & 11.7 & $6407.0 \pm 105.8$ & $1.3 \pm 0.5$ & 4.3 \\
\hline 14091704 & 445.01 & 2.54 & 9.1 & $6307.9 \pm 124.2$ & $1.6 \pm 0.5$ & $4.0 \pm 0.1$ \\
\hline 15445551 & 747.01 & 5.89 & 10.3 & $6271.0 \pm 196.0$ & $1.7 \pm 3.0$ & $4.1 \pm 0.4$ \\
\hline 19519368 & 494.01 & 4.58 & 10.0 & $4985.0 \pm 114.0$ & $1.1 \pm 3.8$ & $4.7 \pm 0.2$ \\
\hline 22221375 & 652.01 & 1.32 & 7.4 & 5903.0 & $1.0 \pm 1.4$ & 4.4 \\
\hline 22529346 & 495.01 & 3.84 & 10.0 & 6459.0 & $1.5 \pm 0.1$ & 4.2 \\
\hline 23434737 & 1203.01 & 1.54 & 8.0 & $5742.2 \pm 30.4$ & $1.4 \pm 0.7$ & $4.3 \pm 0.1$ \\
\hline 25155310 & 114.01 & 3.56 & 10.6 & 5800.0 & $1.3 \pm 0.1$ & 4.3 \\
\hline 29831208 & 124.01 & 3.48 & 11.0 & $5080.0 \pm 181.0$ & $0.9 \pm 9.3$ & $4.5 \pm 2.0$ \\
\hline 29960109 & 393.01 & 6.14 & 10.7 & $3856.0 \pm 68.0$ & $0.6 \pm 0.3$ & $4.7 \pm 0.3$ \\
\hline 30037565 & 1209.01 & 6.01 & 9.6 & $5914.0 \pm 40.3$ & $1.9 \pm 1.9$ & $4.0 \pm 0.1$ \\
\hline 30853470 & 807.01 & 4.9 & 11.2 & $4662.0 \pm 174.0$ & $0.8 \pm 2.9$ & $4.6 \pm 0.2$ \\
\hline 31553893 & 1058.01 & 4.08 & 9.3 & $5785.0 \pm 189.0$ & $1.1 \pm 3.4$ & $4.3 \pm 2.0$ \\
\hline 33692729 & 469.01 & 1.22 & 8.7 & $6114.0 \pm 194.0$ & $1.0 \pm 0.5$ & $4.5 \pm 0.3$ \\
\hline 37575651 & 568.01 & 2.2 & 8.3 & 5780.0 & $1.0 \pm 1.1$ & 4.4 \\
\hline 37749396 & 260.01 & 5.68 & 8.4 & $4111.0 \pm 171.0$ & $0.6 \pm 1.5$ & $4.6 \pm 0.4$ \\
\hline 37770169 & 470.01 & 2.66 & 10.6 & $5418.0 \pm 185.0$ & $0.8 \pm 1.1$ & $4.6 \pm 0.3$ \\
\hline 38696105 & 281.01 & 2.28 & 10.5 & $6002.2 \pm 42.2$ & $1.5 \pm 3.5$ & $4.1 \pm 0.1$ \\
\hline 41227743 & 804.01 & 3.52 & 10.7 & $5988.0 \pm 192.0$ & $1.2 \pm 1.6$ & $4.3 \pm 2.0$ \\
\hline 42054565 & 280.01 & 3.54 & 10.0 & $5454.0 \pm 185.0$ & $0.8 \pm 4.8$ & $4.6 \pm 0.3$ \\
\hline 43647325 & 423.01 & 3.63 & 10.4 & $5904.0 \pm 156.8$ & $1.1 \pm 0.8$ & 4.4 \\
\hline 47384844 & 1022.01 & 4.87 & 8.7 & $6084.0 \pm 111.0$ & $1.4 \pm 0.8$ & $4.1 \pm 0.3$ \\
\hline 47911178 & 471.01 & 2.73 & 9.8 & 6400.0 & $1.3 \pm 0.1$ & 4.3 \\
\hline 48476907 & 658.01 & 2.53 & 9.4 & $6521.0 \pm 199.0$ & $1.5 \pm 19.9$ & $4.2 \pm 2.0$ \\
\hline 48476908 & 659.01 & 2.49 & 9.5 & $5990.0 \pm 192.0$ & $1.7 \pm 17.9$ & $4.0 \pm 0.7$ \\
\hline 49687222 & 254.01 & 5.37 & 9.8 & $6101.0 \pm 193.0$ & $1.0 \pm 4.5$ & $4.5 \pm 0.4$ \\
\hline 49899799 & 416.01 & 3.41 & 8.3 & $6084.8 \pm 35.2$ & $1.9 \pm 0.2$ & $4.0 \pm 0.3$ \\
\hline 50309953 & 1109.01 & 2.14 & 9.8 & $5317.0 \pm 65.7$ & $0.9 \pm 2.7$ & $4.5 \pm 0.1$ \\
\hline 50312495 & 1211.01 & 2.62 & 9.8 & $5572.9 \pm 104.0$ & $1.2 \pm 2.2$ & $3.8 \pm 0.2$ \\
\hline 50618703 & 544.01 & 3.84 & 9.6 & $4665.0 \pm 177.0$ & $0.7 \pm 0.4$ & $4.7 \pm 0.3$ \\
\hline 52204645 & 209.01 & 6.34 & 10.8 & $4892.0 \pm 179.0$ & $0.8 \pm 4.1$ & $4.5 \pm 2.0$ \\
\hline 53189332 & 660.01 & 3.75 & 11.0 & 6055.0 & $1.4 \pm 0.3$ & 4.2 \\
\hline 53735810 & 661.01 & 4.3 & 11.2 & 6580.0 & $1.8 \pm 0.3$ & 4.1 \\
\hline 54085154 & 662.01 & 2.56 & 8.2 & $6380.0 \pm 80.0$ & $1.3 \pm 0.5$ & 4.4 \\
\hline 55559618 & 695.01 & 5.45 & 10.7 & $5591.0 \pm 187.0$ & $1.1 \pm 3.4$ & $4.4 \pm 2.0$ \\
\hline 61538902 & 752.01 & 6.32 & 11.3 & 5900.0 & $0.9 \pm 0.2$ & 4.5 \\
\hline 70513361 & 262.01 & 0.76 & 8.1 & $5302.6 \pm 21.0$ & $0.8 \pm 0.7$ & 4.5 \\
\hline 70914192 & 427.01 & 3.34 & 10.3 & $5271.7 \pm 43.7$ & $1.1 \pm 4.3$ & $4.3 \pm 0.4$ \\
\hline 73228647 & 755.01 & 2.82 & 9.5 & $6003.0 \pm 192.0$ & $1.0 \pm 0.8$ & $4.5 \pm 0.3$ \\
\hline 76989773 & 182.01 & 2.62 & 9.6 & $5569.0 \pm 187.0$ & $1.1 \pm 5.7$ & $4.3 \pm 0.3$ \\
\hline 89020549 & 132.01 & 4.81 & 10.8 & $5673.0 \pm 188.0$ & $0.9 \pm 1.6$ & $4.6 \pm 0.4$ \\
\hline 94986319 & 421.01 & 4.01 & 9.2 & $5718.0 \pm 189.0$ & $1.1 \pm 6.4$ & $4.4 \pm 2.0$ \\
\hline 96097215 & 728.01 & 4.47 & 10.3 & $5272.0 \pm 183.0$ & $0.9 \pm 4.7$ & $4.4 \pm 2.0$ \\
\hline 100100827 & 185.01 & 2.44 & 8.8 & 6400.0 & 1.2 & 4.4 \\
\hline 101230735 & 1060.01 & 3.83 & 9.6 & $5687.9 \pm 76.0$ & $1.3 \pm 6.0$ & $4.6 \pm 0.1$ \\
\hline 101955023 & 667.01 & 4.14 & 10.9 & 3202.0 & $0.3 \pm 0.8$ & 4.9 \\
\hline 103633434 & 1235.01 & 2.69 & 9.9 & $3912.0 \pm 157.0$ & $0.6 \pm 0.2$ & 4.6 \\
\hline 106402532 & 733.01 & 4.2 & 8.8 & $5969.0 \pm 192.0$ & $1.0 \pm 1.1$ & $4.5 \pm 0.3$ \\
\hline 111991770 & 820.01 & 4.8 & 10.4 & 6300.0 & $1.5 \pm 0.1$ & 4.2 \\
\hline 116483734 & 1412.01 & 6.46 & 11.1 & $4223.4 \pm 133.4$ & $0.7 \pm 11.3$ & $4.6 \pm 0.1$ \\
\hline 117979897 & 443.01 & 6.05 & 12.0 & $5928.0 \pm 114.4$ & $1.4 \pm 0.5$ & 4.3 \\
\hline 119700084 & 1413.01 & 4.77 & 10.1 & $5427.0 \pm 141.3$ & $0.9 \pm 8.7$ & $4.5 \pm 0.1$ \\
\hline 120602501 & 1366.01 & 3.07 & 8.5 & $5705.0 \pm 185.1$ & $1.7 \pm 5.4$ & $4.0 \pm 0.1$ \\
\hline 120610833 & 229.01 & 6.0 & 11.4 & 5100.0 & $0.9 \pm 0.2$ & 4.5 \\
\hline 120960812 & 1237.01 & 3.49 & 10.3 & $6212.0 \pm 132.8$ & $1.5 \pm 4.1$ & $4.2 \pm 0.1$ \\
\hline 122612091 & 264.01 & 1.9 & 10.5 & 6250.0 & $2.0 \pm 0.1$ & 4.0 \\
\hline 123702439 & 499.01 & 1.9 & 10.1 & $5997.0 \pm 192.0$ & $1.0 \pm 1.6$ & $4.5 \pm 0.3$ \\
\hline 124573851 & 669.01 & 2.81 & 10.1 & $5581.9 \pm 76.0$ & $1.2 \pm 0.6$ & $4.1 \pm 0.1$ \\
\hline 126733133 & 570.01 & 5.39 & 10.0 & $5973.0 \pm 192.0$ & $1.3 \pm 4.4$ & $4.3 \pm 0.3$ \\
\hline 127530399 & 822.01 & 6.41 & 11.1 & 4775.0 & $0.7 \pm 0.2$ & 4.6 \\
\hline 128790976 & 1124.01 & 1.86 & 7.2 & $6541.0 \pm 94.0$ & $1.6 \pm 4.7$ & $4.2 \pm 0.1$ \\
\hline 131081852 & 758.01 & 4.91 & 9.9 & $6072.0 \pm 193.0$ & $1.3 \pm 19.4$ & $4.3 \pm 2.0$ \\
\hline 131419878 & 720.01 & 1.15 & 8.8 & $6482.3 \pm 205.2$ & $1.5 \pm 0.3$ & $4.2 \pm 0.4$ \\
\hline 134537478 & 501.01 & 4.55 & 10.4 & 5720.0 & $1.5 \pm 0.1$ & 4.2 \\
\hline 138588540 & 1434.01 & 5.57 & 8.1 & $5393.9 \pm 139.7$ & $0.7 \pm 0.9$ & $4.7 \pm 0.1$ \\
\hline 140068425 & 140.01 & 6.47 & 9.8 & $6174.0 \pm 194.0$ & $1.3 \pm 5.1$ & $4.3 \pm 2.0$ \\
\hline 140760434 & 1229.01 & 5.38 & 10.7 & $6028.8 \pm 44.1$ & $2.0 \pm 3.8$ & $4.0 \pm 0.1$ \\
\hline
\end{tabular}


A\&A 639, A35 (2020)

Table C.2. continued.

\begin{tabular}{|c|c|c|c|c|c|c|}
\hline TIC ID & Full TOI id & $\begin{array}{c}\text { Peak-to-peak light curve } \\
{[\mathrm{ppt}]}\end{array}$ & TESS mag & $\begin{array}{l}T_{\text {eff }} \\
{[\mathrm{K}]}\end{array}$ & $\begin{array}{c}R_{\text {star }} \\
{\left[R_{\text {Sun }}\right]}\end{array}$ & $\begin{array}{c}\log g \\
{\left[\mathrm{~cm} \mathrm{~s}^{-2}\right]}\end{array}$ \\
\hline 141527965 & 1216.01 & 5.87 & 11.4 & $4280.0 \pm 171.0$ & $0.7 \pm 0.4$ & $4.6 \pm 0.2$ \\
\hline 143350972 & 440.01 & 1.27 & 7.7 & $5759.2 \pm 38.2$ & $1.0 \pm 0.1$ & 4.4 \\
\hline 144065872 & 105.01 & 4.56 & 9.5 & 5630.0 & $1.1 \pm 0.1$ & 4.4 \\
\hline 147950620 & 1194.01 & 3.25 & 10.5 & $5339.9 \pm 155.2$ & $1.0 \pm 0.5$ & $4.4 \pm 0.1$ \\
\hline 148782377 & 1415.01 & 2.84 & 8.4 & $6383.0 \pm 130.4$ & $1.4 \pm 0.6$ & $4.2 \pm 0.1$ \\
\hline 149301575 & 809.01 & 4.56 & 10.3 & $5804.3 \pm 76.0$ & $1.3 \pm 1.0$ & $3.9 \pm 0.1$ \\
\hline 149603524 & 102.01 & 2.37 & 9.7 & 6280.0 & 1.3 & 4.3 \\
\hline 150030205 & 286.01 & 1.72 & 9.1 & $5245.0 \pm 183.0$ & $0.8 \pm 1.7$ & $4.6 \pm 0.3$ \\
\hline 150098860 & 220.01 & 5.38 & 9.7 & $5272.7 \pm 45.6$ & $0.9 \pm 1.9$ & $4.0 \pm 0.1$ \\
\hline 153065527 & 406.01 & 4.14 & 11.2 & $3349.0 \pm 63.0$ & $0.3 \pm 0.7$ & $4.9 \pm 0.4$ \\
\hline 153976959 & 1435.01 & 4.15 & 10.0 & $5142.0 \pm 122.7$ & $0.8 \pm 2.4$ & $4.5 \pm 0.1$ \\
\hline 154089169 & 1174.01 & 5.29 & 10.3 & $5029.5 \pm 59.9$ & $0.8 \pm 0.2$ & $4.6 \pm 0.1$ \\
\hline 154383539 & 1436.01 & 3.86 & 11.1 & $5011.2 \pm 102.7$ & $0.7 \pm 1.9$ & $4.6 \pm 0.1$ \\
\hline 154840461 & 1153.01 & 2.35 & 8.4 & $6528.3 \pm 179.4$ & $1.7 \pm 0.5$ & $4.2 \pm 0.1$ \\
\hline 158002130 & 1180.01 & 2.55 & 10.1 & $4738.1 \pm 139.3$ & $0.7 \pm 0.2$ & $4.6 \pm 0.1$ \\
\hline 158025009 & 1416.01 & 2.17 & 9.1 & $4946.0 \pm 128.6$ & $0.8 \pm 1.8$ & $4.5 \pm 0.1$ \\
\hline 158623531 & 246.01 & 3.55 & 11.2 & 5070.0 & $0.9 \pm 0.1$ & 4.5 \\
\hline 158978373 & 823.01 & 2.91 & 10.0 & $6309.0 \pm 196.0$ & $1.4 \pm 1.1$ & $4.2 \pm 0.3$ \\
\hline 159510109 & 1141.01 & 6.31 & 8.9 & $5894.7 \pm 90.3$ & $1.1 \pm 0.3$ & $4.4 \pm 0.1$ \\
\hline 159951311 & 265.01 & 4.21 & 11.7 & 5310.0 & $0.8 \pm 0.2$ & 4.6 \\
\hline 160074939 & 230.01 & 1.79 & 8.7 & $6467.0 \pm 198.0$ & $1.6 \pm 1.6$ & $4.2 \pm 0.3$ \\
\hline 160148385 & 247.01 & 4.87 & 11.9 & 5540.0 & $1.0 \pm 0.2$ & 4.4 \\
\hline 166739520 & 190.01 & 1.56 & 9.6 & 6038.0 & $1.2 \pm 0.1$ & 4.4 \\
\hline 166836920 & 267.01 & 1.27 & 8.9 & 6180.0 & $1.8 \pm 0.1$ & 4.1 \\
\hline 167303382 & 802.01 & 1.56 & 7.5 & $5235.8 \pm 28.6$ & $0.8 \pm 0.4$ & 4.4 \\
\hline 167342439 & 707.01 & 5.55 & 10.1 & $5409.2 \pm 41.8$ & $1.0 \pm 1.7$ & $4.5 \pm 0.1$ \\
\hline 167415965 & 214.01 & 1.73 & 8.0 & $5346.2 \pm 12.9$ & $0.9 \pm 0.4$ & 4.5 \\
\hline 167754523 & 409.01 & 4.84 & 9.1 & $4913.8 \pm 71.5$ & $0.8 \pm 0.1$ & $4.5 \pm 0.2$ \\
\hline 170102285 & 477.01 & 5.05 & 11.7 & 5150.0 & $0.8 \pm 0.1$ & 4.6 \\
\hline 172193428 & 502.01 & 3.76 & 10.3 & $5642.0 \pm 188.0$ & $1.4 \pm 1.5$ & $4.2 \pm 0.3$ \\
\hline 175180796 & 816.01 & 4.7 & 11.1 & $4146.6 \pm 148.6$ & $0.6 \pm 1.1$ & $4.6 \pm 0.1$ \\
\hline 176778112 & 408.01 & 5.84 & 9.6 & $5600.0 \pm 187.0$ & $1.0 \pm 5.9$ & $4.4 \pm 0.3$ \\
\hline 177258735 & 801.01 & 1.34 & 7.5 & $6283.4 \pm 44.4$ & $1.6 \pm 0.4$ & 4.4 \\
\hline 179317684 & 163.01 & 3.55 & 10.8 & $6387.9 \pm 196.4$ & $1.7 \pm 1.0$ & $4.2 \pm 2.0$ \\
\hline 183120439 & 169.01 & 6.23 & 11.7 & $5780.6 \pm 401.1$ & $1.3 \pm 1.5$ & $4.3 \pm 2.0$ \\
\hline 183537452 & 192.01 & 2.65 & 10.2 & 4800.0 & $0.8 \pm 0.1$ & 4.5 \\
\hline 183985250 & 193.01 & 4.66 & 9.1 & $5422.0 \pm 185.0$ & $1.0 \pm 1.4$ & $4.4 \pm 0.3$ \\
\hline 184952758 & 719.01 & 2.59 & 9.0 & $5761.2 \pm 89.8$ & $0.9 \pm 0.1$ & $4.5 \pm 0.3$ \\
\hline 188768068 & 1462.01 & 0.72 & 5.9 & $5801.9 \pm 124.6$ & $1.3 \pm 1.3$ & $4.3 \pm 0.1$ \\
\hline 190990336 & 585.01 & 3.74 & 8.9 & $6118.1 \pm 137.4$ & $1.7 \pm 0.9$ & $4.3 \pm 0.3$ \\
\hline 198212955 & 1242.01 & 4.35 & 11.6 & $4255.0 \pm 128.2$ & $0.7 \pm 2.8$ & $4.6 \pm 0.1$ \\
\hline 198241702 & 1269.01 & 3.21 & 10.9 & $5591.0 \pm 185.0$ & $0.8 \pm 0.4$ & 4.4 \\
\hline 198356533 & 1437.01 & 2.39 & 8.7 & $6093.0 \pm 124.7$ & $1.2 \pm 0.7$ & $4.3 \pm 0.1$ \\
\hline 198390247 & 1453.01 & 2.31 & 10.1 & $4920.0 \pm 127.0$ & $0.7 \pm 1.6$ & $4.6 \pm 0.1$ \\
\hline 199688472 & 1292.01 & 3.65 & 9.8 & $5672.9 \pm 168.9$ & $1.5 \pm 6.6$ & $4.1 \pm 0.1$ \\
\hline 200807066 & 869.01 & 2.93 & 8.9 & $5908.0 \pm 191.0$ & $1.2 \pm 2.6$ & $4.3 \pm 0.3$ \\
\hline 201793781 & 248.01 & 2.8 & 8.4 & $5711.7 \pm 57.0$ & $1.2 \pm 0.7$ & $3.8 \pm 0.1$ \\
\hline 206466531 & 410.01 & 2.47 & 10.0 & $6000.2 \pm 130.8$ & $1.5 \pm 0.2$ & $4.4 \pm 0.1$ \\
\hline 207081058 & 121.01 & 4.51 & 9.9 & $6053.0 \pm 193.0$ & $1.2 \pm 2.2$ & $4.3 \pm 0.3$ \\
\hline 207084429 & 381.01 & 0.78 & 7.2 & $5883.0 \pm 52.4$ & $1.9 \pm 0.2$ & - \\
\hline 211438925 & 194.01 & 3.69 & 10.2 & 5940.0 & $1.4 \pm 0.1$ & 4.2 \\
\hline 219239945 & 701.01 & 2.74 & 10.9 & $5837.0 \pm 190.0$ & $1.1 \pm 0.7$ & $4.4 \pm 0.3$ \\
\hline 219338557 & 133.01 & 4.54 & 9.9 & $4028.0 \pm 170.0$ & $0.7 \pm 1.9$ & $4.6 \pm 0.2$ \\
\hline 219379012 & 397.01 & 4.52 & 10.2 & $5973.5 \pm 185.9$ & $1.0 \pm 2.5$ & $3.9 \pm 0.1$ \\
\hline 219388773 & 399.01 & 5.22 & 10.6 & $4752.8 \pm 64.0$ & $1.0 \pm 0.5$ & $4.4 \pm 0.1$ \\
\hline 219698950 & 766.01 & 5.49 & 11.5 & 5870.0 & $1.0 \pm 0.1$ & 4.4 \\
\hline 219850915 & 1244.01 & 5.37 & 11.0 & $4599.0 \pm 123.0$ & $0.7 \pm 2.1$ & $4.6 \pm 0.1$ \\
\hline 220396259 & 379.01 & 2.29 & 9.9 & $5825.0 \pm 97.8$ & $1.7 \pm 0.2$ & $4.0 \pm 0.1$ \\
\hline 224297258 & 1279.01 & 2.68 & 10.0 & $5477.0 \pm 120.1$ & $0.8 \pm 2.0$ & $4.6 \pm 0.1$ \\
\hline 229650439 & 1438.01 & 4.09 & 10.3 & $5374.0 \pm 183.0$ & $0.8 \pm 0.3$ & $6.0 \pm 0.3$ \\
\hline 229770036 & 1348.01 & 5.24 & 10.7 & $4567.0 \pm 143.3$ & $0.8 \pm 0.2$ & $4.4 \pm 0.1$ \\
\hline 230017324 & 1280.01 & 4.07 & 10.6 & $4665.0 \pm 128.1$ & $0.7 \pm 2.5$ & $4.6 \pm 0.1$ \\
\hline 230088370 & 1176.01 & 2.2 & 10.2 & $6557.7 \pm 83.3$ & $1.6 \pm 0.4$ & $4.2 \pm 0.1$ \\
\hline 230982885 & 195.01 & 1.58 & 10.0 & 5640.0 & $1.1 \pm 0.1$ & 4.4 \\
\hline 231912935 & 215.01 & 4.17 & 10.5 & $5821.0 \pm 190.0$ & $1.0 \pm 1.7$ & $4.4 \pm 0.3$ \\
\hline 232025086 & 874.01 & 2.15 & 8.8 & $6437.0 \pm 198.0$ & $1.4 \pm 1.3$ & $4.2 \pm 0.3$ \\
\hline 232540264 & 1247.01 & 1.49 & 8.5 & $5711.8 \pm 106.3$ & $1.1 \pm 0.6$ & $4.4 \pm 0.1$ \\
\hline 232612416 & 1248.01 & 3.79 & 11.1 & $5227.0 \pm 119.5$ & $0.9 \pm 0.6$ & $4.5 \pm 0.1$ \\
\hline 232967440 & 1173.01 & 2.39 & 10.3 & $5429.6 \pm 292.5$ & $1.0 \pm 0.5$ & $4.4 \pm 0.1$ \\
\hline 232971294 & 1281.01 & 2.46 & 9.7 & $6191.0 \pm 127.5$ & $1.3 \pm 1.4$ & $4.3 \pm 0.1$ \\
\hline 232976128 & 1249.01 & 2.67 & 10.2 & $5453.0 \pm 117.9$ & $1.0 \pm 2.3$ & $4.4 \pm 0.1$ \\
\hline
\end{tabular}


S. Hojjatpanah et al.: The correlation between photometric variability and radial velocity jitter

Table C.2. continued.

\begin{tabular}{|c|c|c|c|c|c|c|}
\hline TIC ID & Full TOI id & $\begin{array}{c}\text { Peak-to-peak light curve } \\
\text { [ppt] }\end{array}$ & TESS mag & $\begin{array}{l}T_{\text {eff }} \\
{[\mathrm{K}]}\end{array}$ & $\begin{array}{c}R_{\text {star }} \\
{\left[R_{\text {Sun }}\right]}\end{array}$ & $\begin{array}{c}\log g \\
{\left[\mathrm{~cm} \mathrm{~s}^{-2}\right]} \\
\end{array}$ \\
\hline 232982558 & 1439.01 & 1.94 & 10.0 & $5873.0 \pm 123.8$ & $1.6 \pm 0.9$ & $4.0 \pm 0.1$ \\
\hline 233087860 & 1184.01 & 5.62 & 9.9 & $4681.4 \pm 80.5$ & $0.7 \pm 1.1$ & $4.6 \pm 0.1$ \\
\hline 233390838 & 1341.01 & 2.25 & 9.5 & $6477.6 \pm 101.6$ & $1.7 \pm 5.4$ & $4.1 \pm 0.1$ \\
\hline 233541860 & 1486.01 & 2.44 & 10.0 & $4154.0 \pm 173.4$ & $0.6 \pm 0.1$ & $4.5 \pm 0.1$ \\
\hline 233617847 & 1440.01 & 4.07 & 9.6 & 5780.0 & $1.0 \pm 0.9$ & 4.4 \\
\hline 233681149 & 1340.01 & 2.96 & 10.8 & $5929.0 \pm 124.8$ & $1.0 \pm 0.4$ & $4.5 \pm 0.1$ \\
\hline 233684822 & 1253.01 & 2.08 & 9.9 & $5779.0 \pm 188.0$ & $1.5 \pm 3.2$ & $4.9 \pm 0.4$ \\
\hline 233951353 & 1441.01 & 2.02 & 9.5 & $5885.0 \pm 201.6$ & $1.2 \pm 1.4$ & $4.3 \pm 0.1$ \\
\hline 234345288 & 213.01 & 3.34 & 9.7 & $4566.4 \pm 64.0$ & $0.7 \pm 1.1$ & $4.9 \pm 0.1$ \\
\hline 236714379 & 1254.01 & 3.13 & 10.8 & $5451.0 \pm 125.9$ & $1.1 \pm 0.9$ & $4.3 \pm 0.1$ \\
\hline 237222864 & 1255.01 & 3.04 & 9.1 & $5126.0 \pm 124.4$ & $0.9 \pm 0.5$ & $4.5 \pm 0.1$ \\
\hline 237232044 & 1443.01 & 2.52 & 9.9 & $5236.0 \pm 125.3$ & $0.7 \pm 0.5$ & $4.6 \pm 0.1$ \\
\hline 238176110 & 116.01 & 3.61 & 11.0 & 4920.0 & $0.9 \pm 0.1$ & 4.5 \\
\hline 238197638 & 903.01 & 5.37 & 11.7 & $6266.0 \pm 41.0$ & $1.4 \pm 2.0$ & $4.3 \pm 0.2$ \\
\hline 240968774 & 1467.01 & 6.01 & 10.6 & $3834.0 \pm 157.0$ & $0.5 \pm 2.4$ & 4.7 \\
\hline 243200602 & 826.01 & 4.95 & 10.5 & 5625.0 & $1.0 \pm 0.1$ & 4.5 \\
\hline 248075138 & 769.01 & 5.9 & 11.3 & 5315.0 & $0.9 \pm 0.2$ & 4.5 \\
\hline 250386181 & 390.01 & 4.45 & 9.9 & $6321.5 \pm 53.5$ & $1.1 \pm 6.5$ & $4.2 \pm 0.1$ \\
\hline 253990973 & 1061.01 & 4.12 & 9.7 & $5525.0 \pm 186.0$ & $1.2 \pm 0.6$ & $4.3 \pm 0.3$ \\
\hline 255685030 & 799.01 & 4.42 & 10.5 & $5643.0 \pm 188.0$ & $1.0 \pm 3.5$ & $4.5 \pm 0.3$ \\
\hline 257567854 & 403.01 & 6.04 & 11.2 & 6153.0 & $1.2 \pm 0.2$ & 4.3 \\
\hline 258514800 & 1444.01 & 4.17 & 10.2 & $5466.0 \pm 121.5$ & $0.9 \pm 3.4$ & $4.5 \pm 0.1$ \\
\hline 258777137 & 1183.01 & 4.93 & 9.9 & $5095.6 \pm 1241.5$ & $1.1 \pm 0.2$ & - \\
\hline 259511357 & 271.01 & 2.53 & 8.4 & $6025.0 \pm 192.0$ & $1.2 \pm 1.5$ & $4.3 \pm 0.4$ \\
\hline 259592689 & 429.01 & 2.74 & 10.2 & $5160.7 \pm 90.5$ & $1.0 \pm 0.4$ & $4.4 \pm 2.0$ \\
\hline 259701242 & 401.01 & 3.79 & 8.2 & $5928.0 \pm 85.6$ & $1.0 \pm 0.1$ & $4.5 \pm 0.2$ \\
\hline 260043723 & 217.01 & 4.65 & 8.7 & $6278.0 \pm 196.0$ & $1.7 \pm 1.3$ & $4.1 \pm 0.3$ \\
\hline 260708537 & 486.01 & 3.47 & 9.3 & $3445.0 \pm 87.0$ & $0.4 \pm 1.0$ & $4.9 \pm 0.1$ \\
\hline 261257684 & 904.01 & 5.75 & 10.8 & $3992.0 \pm 170.0$ & $0.6 \pm 1.0$ & $4.6 \pm 0.2$ \\
\hline 261867566 & 905.01 & 5.65 & 10.5 & $5565.0 \pm 187.0$ & $1.0 \pm 1.8$ & $4.5 \pm 0.3$ \\
\hline 263003176 & 130.01 & 1.79 & 7.4 & $6295.7 \pm 56.5$ & $1.1 \pm 1.1$ & 4.4 \\
\hline 264979636 & 518.01 & 5.27 & 10.1 & $5596.0 \pm 187.0$ & $1.1 \pm 4.2$ & $4.3 \pm 0.3$ \\
\hline 266980320 & 118.01 & 1.68 & 9.2 & $5586.0 \pm 187.0$ & $1.1 \pm 0.6$ & $4.4 \pm 0.3$ \\
\hline 268644785 & 505.01 & 2.85 & 10.7 & 6003.0 & $1.5 \pm 0.1$ & 4.2 \\
\hline 270341214 & 173.01 & 1.52 & 8.9 & $6409.0 \pm 197.0$ & $1.5 \pm 1.3$ & $4.2 \pm 0.3$ \\
\hline 271168962 & 828.01 & 6.22 & 9.4 & 6030.0 & $1.5 \pm 0.1$ & 4.1 \\
\hline 271893367 & 150.01 & 3.26 & 10.9 & $5766.0 \pm 37.3$ & $1.6 \pm 0.3$ & $4.1 \pm 0.1$ \\
\hline 271900960 & 389.01 & 2.91 & 8.3 & $6457.7 \pm 115.5$ & $1.4 \pm 2.6$ & $4.4 \pm 0.3$ \\
\hline 272635712 & 1349.01 & 2.25 & 9.3 & $5711.1 \pm 365.0$ & $1.3 \pm 0.4$ & $4.2 \pm 0.1$ \\
\hline 273985865 & 1208.01 & 3.83 & 10.5 & $5626.0 \pm 188.0$ & $0.9 \pm 2.3$ & $4.6 \pm 0.3$ \\
\hline 274138511 & 506.01 & 3.15 & 10.4 & $5280.0 \pm 183.0$ & $0.9 \pm 3.1$ & $4.4 \pm 2.0$ \\
\hline 277103955 & 284.01 & 3.21 & 9.2 & $4779.8 \pm 128.6$ & $0.7 \pm 0.1$ & $4.6 \pm 0.3$ \\
\hline 277507814 & 1286.01 & 4.42 & 10.2 & $6588.0 \pm 247.6$ & $1.6 \pm 3.1$ & $4.2 \pm 0.1$ \\
\hline 277683130 & 138.01 & 5.77 & 9.5 & $5722.0 \pm 189.0$ & $1.1 \pm 8.3$ & $4.4 \pm 2.0$ \\
\hline 279425357 & 739.01 & 6.5 & 11.5 & $6083.6 \pm 125.0$ & $1.2 \pm 2.6$ & $4.3 \pm 0.2$ \\
\hline 280095254 & 235.01 & 2.79 & 9.3 & $5454.4 \pm 77.3$ & $1.0 \pm 5.8$ & $3.4 \pm 0.1$ \\
\hline 280206394 & 677.01 & 4.18 & 9.2 & $6446.0 \pm 198.0$ & $1.2 \pm 1.5$ & $4.4 \pm 0.3$ \\
\hline 280830734 & 188.01 & 2.48 & 9.4 & $6340.3 \pm 204.0$ & $1.6 \pm 7.5$ & $4.2 \pm 0.3$ \\
\hline 281575427 & 205.01 & 2.2 & 9.4 & $6222.5 \pm 83.0$ & $1.5 \pm 2.7$ & $4.1 \pm 0.2$ \\
\hline 286132427 & 635.01 & 4.94 & 8.1 & $5914.0 \pm 191.0$ & $1.0 \pm 0.9$ & $4.5 \pm 0.3$ \\
\hline 287156968 & 1230.01 & 1.69 & 9.6 & $5696.0 \pm 188.0$ & $1.2 \pm 1.1$ & $4.3 \pm 0.3$ \\
\hline 288132261 & 1258.01 & 5.71 & 9.1 & $6124.0 \pm 126.6$ & $1.5 \pm 0.4$ & $4.2 \pm 0.1$ \\
\hline 290131778 & 123.01 & 1.22 & 8.8 & $6188.0 \pm 194.0$ & $1.3 \pm 7.7$ & $4.3 \pm 2.0$ \\
\hline 290348383 & 1099.01 & 4.49 & 7.4 & $4867.0 \pm 179.0$ & $1.0 \pm 0.6$ & 4.4 \\
\hline 294301883 & 774.01 & 6.17 & 11.3 & 6070.0 & $1.1 \pm 0.1$ & 4.4 \\
\hline 294471966 & 1446.01 & 5.04 & 10.8 & $5180.0 \pm 101.3$ & $0.8 \pm 2.1$ & $4.5 \pm 0.1$ \\
\hline 294781547 & 1218.01 & 3.68 & 10.3 & $5456.0 \pm 48.9$ & $1.0 \pm 2.9$ & $4.5 \pm 0.1$ \\
\hline 294981566 & 1219.01 & 6.24 & 10.1 & $6734.0 \pm 202.0$ & $1.6 \pm 2.1$ & $4.2 \pm 0.4$ \\
\hline 299799658 & 1062.01 & 4.04 & 9.4 & $5394.0 \pm 185.0$ & $0.9 \pm 0.5$ & $4.5 \pm 0.3$ \\
\hline 300293197 & 211.01 & 5.7 & 9.4 & $5874.0 \pm 102.6$ & $1.1 \pm 6.4$ & $4.4 \pm 0.1$ \\
\hline 304042899 & 775.01 & 2.71 & 9.5 & $6746.0 \pm 202.0$ & $1.2 \pm 8.5$ & $4.4 \pm 0.4$ \\
\hline 306362738 & 479.01 & 2.9 & 10.8 & 5600.0 & $1.0 \pm 0.1$ & 4.4 \\
\hline 309791156 & 533.01 & 5.76 & 10.9 & $4666.1 \pm 64.0$ & $1.1 \pm 1.7$ & $4.2 \pm 0.1$ \\
\hline 314865962 & 208.01 & 3.96 & 10.3 & $5493.0 \pm 186.0$ & $1.0 \pm 1.3$ & $4.4 \pm 0.3$ \\
\hline 317060587 & 1052.01 & 2.74 & 9.0 & $5958.2 \pm 76.0$ & $1.6 \pm 4.5$ & $4.3 \pm 0.1$ \\
\hline 317548889 & 480.01 & 1.05 & 6.8 & $6212.6 \pm 49.0$ & $1.6 \pm 1.8$ & 4.4 \\
\hline 320004517 & 1055.01 & 2.73 & 8.1 & $5783.5 \pm 7.8$ & $1.1 \pm 0.6$ & 4.5 \\
\hline 321857016 & 1420.01 & 4.48 & 11.2 & $5387.0 \pm 136.6$ & $0.9 \pm 0.7$ & $4.5 \pm 0.1$ \\
\hline 322063810 & 253.01 & 1.78 & 9.3 & $4020.0 \pm 170.0$ & $0.6 \pm 1.6$ & 4.4 \\
\hline 325680697 & 414.01 & 3.1 & 9.1 & $5773.7 \pm 76.0$ & $1.3 \pm 5.3$ & $3.5 \pm 0.1$ \\
\hline
\end{tabular}


A\&A 639, A35 (2020)

Table C.2. continued.

\begin{tabular}{|c|c|c|c|c|c|c|}
\hline TIC ID & Full TOI id & $\begin{array}{c}\text { Peak-to-peak light curve } \\
\text { [ppt] }\end{array}$ & TESS mag & $\begin{array}{l}T_{\text {eff }} \\
{[\mathrm{K}]}\end{array}$ & $\begin{array}{c}R_{\text {star }} \\
{\left[R_{\text {Sun }}\right]}\end{array}$ & $\begin{array}{c}\log g \\
{\left[\mathrm{~cm} \mathrm{~s}^{-2}\right]}\end{array}$ \\
\hline 327301957 & 1074.01 & 2.9 & 10.6 & $5206.0 \pm 183.0$ & $0.8 \pm 0.5$ & $4.6 \pm 0.3$ \\
\hline 335499997 & 680.01 & 5.44 & 9.0 & $5967.0 \pm 192.0$ & $1.8 \pm 1.4$ & $3.9 \pm 0.4$ \\
\hline 339672028 & 481.01 & 4.4 & 9.4 & $5760.0 \pm 189.0$ & $1.7 \pm 1.6$ & $4.0 \pm 0.3$ \\
\hline 339733013 & 417.01 & 4.24 & 10.0 & $5834.2 \pm 179.5$ & $1.3 \pm 0.3$ & $4.2 \pm 0.1$ \\
\hline 339857675 & 686.01 & 2.26 & 9.9 & $6279.8 \pm 127.2$ & $1.6 \pm 0.3$ & $4.3 \pm 0.4$ \\
\hline 346418409 & 1423.01 & 5.63 & 10.9 & $4993.0 \pm 127.1$ & $0.7 \pm 7.7$ & $4.7 \pm 0.1$ \\
\hline 348844154 & 916.01 & 5.23 & 11.2 & $5493.2 \pm 126.6$ & $1.9 \pm 1.2$ & $3.9 \pm 0.5$ \\
\hline 349827430 & 1148.01 & 1.51 & 7.9 & $6403.9 \pm 184.1$ & $1.5 \pm 0.9$ & $4.2 \pm 0.1$ \\
\hline 350153977 & 908.01 & 3.68 & 10.6 & $5500.0 \pm 76.0$ & $1.1 \pm 3.9$ & $5.0 \pm 0.1$ \\
\hline 350584963 & 787.01 & 3.81 & 9.5 & $6623.0 \pm 200.0$ & $1.3 \pm 2.3$ & $4.4 \pm 0.3$ \\
\hline 350743714 & 165.01 & 2.67 & 9.8 & $6038.0 \pm 54.0$ & $1.9 \pm 2.1$ & $4.0 \pm 0.1$ \\
\hline 352413427 & 1473.01 & 2.73 & 8.3 & $5958.0 \pm 121.3$ & $1.0 \pm 0.5$ & $4.5 \pm 0.1$ \\
\hline 352764091 & 1287.01 & 1.81 & 8.6 & $5891.0 \pm 124.6$ & $1.2 \pm 1.7$ & $4.3 \pm 0.1$ \\
\hline 354594208 & 1492.01 & 3.45 & 9.8 & $5904.0 \pm 249.1$ & $1.2 \pm 0.4$ & $4.3 \pm 0.1$ \\
\hline 358460246 & 867.01 & 4.07 & 10.4 & $5777.4 \pm 113.6$ & $1.2 \pm 1.0$ & $4.3 \pm 0.2$ \\
\hline 359271092 & 741.01 & 5.51 & 7.7 & $3766.0 \pm 95.0$ & $0.5 \pm 0.7$ & $4.8 \pm 0.1$ \\
\hline 364107753 & 909.01 & 1.0 & 7.0 & $6059.1 \pm 50.4$ & $1.6 \pm 0.8$ & $4.3 \pm 0.1$ \\
\hline 364186197 & 1408.01 & 2.4 & 8.8 & $6594.0 \pm 90.9$ & $1.6 \pm 0.6$ & $4.2 \pm 0.2$ \\
\hline 364393429 & 1207.01 & 2.92 & 9.2 & $6282.0 \pm 196.0$ & $1.2 \pm 1.0$ & $4.4 \pm 0.3$ \\
\hline 365733349 & 1288.01 & 3.15 & 9.9 & $6180.0 \pm 362.0$ & $0.9 \pm 0.4$ & $4.6 \pm 0.1$ \\
\hline 366989877 & 1054.01 & 1.64 & 8.4 & $6122.0 \pm 194.0$ & $1.2 \pm 0.9$ & $4.3 \pm 0.3$ \\
\hline 369327947 & 910.01 & 4.38 & 10.3 & $3194.0 \pm 101.0$ & $0.3 \pm 1.3$ & $5.0 \pm 0.1$ \\
\hline 373844472 & 275.01 & 4.27 & 11.0 & $5616.0 \pm 187.0$ & $1.1 \pm 18.7$ & $4.4 \pm 2.0$ \\
\hline 374829238 & 785.01 & 6.3 & 11.5 & 3783.0 & $1.0 \pm 0.2$ & 4.4 \\
\hline 374997123 & 1222.01 & 3.37 & 10.3 & $6082.0 \pm 193.0$ & $1.2 \pm 2.4$ & $4.3 \pm 0.3$ \\
\hline 375059587 & 786.01 & 2.54 & 9.8 & $5853.8 \pm 45.2$ & $1.4 \pm 1.2$ & $4.2 \pm 0.1$ \\
\hline 377293776 & 1450.01 & 3.14 & 10.0 & $3407.0 \pm 157.0$ & $0.5 \pm 2.6$ & 4.8 \\
\hline 382188882 & 276.01 & 3.75 & 10.6 & $6366.0 \pm 197.0$ & $1.5 \pm 5.3$ & $4.2 \pm 0.5$ \\
\hline 382437043 & 1223.01 & 4.81 & 9.3 & $6516.0 \pm 199.0$ & $1.9 \pm 3.0$ & $4.0 \pm 0.4$ \\
\hline 382474101 & 349.01 & 4.08 & 10.2 & $6054.0 \pm 193.0$ & $1.2 \pm 14.0$ & $4.3 \pm 2.0$ \\
\hline 382626661 & 283.01 & 5.09 & 9.6 & $5250.0 \pm 183.0$ & $0.7 \pm 0.9$ & $4.6 \pm 0.3$ \\
\hline 386435344 & 575.01 & 2.4 & 8.7 & $6529.0 \pm 199.0$ & $1.9 \pm 13.5$ & $4.0 \pm 0.3$ \\
\hline 387259626 & 1455.01 & 3.87 & 10.2 & $6458.8 \pm 157.5$ & $1.4 \pm 0.9$ & $4.3 \pm 0.1$ \\
\hline 391949880 & 128.01 & 1.79 & 7.9 & $6086.0 \pm 193.0$ & $1.3 \pm 0.9$ & $4.3 \pm 0.3$ \\
\hline 393414358 & 483.01 & 2.35 & 10.4 & 5570.0 & $1.9 \pm 0.2$ & 4.0 \\
\hline 394657039 & 159.01 & 3.44 & 10.5 & $6404.0 \pm 463.3$ & $1.6 \pm 3.1$ & $4.4 \pm 0.2$ \\
\hline 394698182 & 170.01 & 6.4 & 11.9 & $5768.2 \pm 56.4$ & $1.2 \pm 0.4$ & $4.3 \pm 2.0$ \\
\hline 399144800 & 1213.01 & 3.59 & 10.6 & $6270.0 \pm 196.0$ & $1.0 \pm 1.9$ & $4.5 \pm 0.4$ \\
\hline 402026209 & 232.01 & 5.69 & 11.8 & 5436.0 & $0.9 \pm 0.1$ & 4.5 \\
\hline 403224672 & 141.01 & 1.19 & 7.4 & $5795.3 \pm 44.6$ & $1.2 \pm 0.4$ & 4.4 \\
\hline 406941612 & 912.01 & 3.81 & 10.4 & $3566.0 \pm 66.0$ & $0.4 \pm 0.4$ & $4.8 \pm 0.4$ \\
\hline 407126408 & 913.01 & 5.4 & 9.6 & $4948.0 \pm 180.0$ & $0.7 \pm 1.0$ & $4.6 \pm 0.3$ \\
\hline 407966340 & 554.01 & 1.7 & 6.4 & $6337.9 \pm 44.7$ & $1.8 \pm 0.9$ & 4.4 \\
\hline 408159788 & 420.01 & 3.67 & 10.6 & $5747.3 \pm 518.8$ & $0.8 \pm 0.4$ & $4.6 \pm 0.3$ \\
\hline 408310006 & 576.01 & 5.23 & 8.8 & $6313.0 \pm 196.0$ & $1.3 \pm 0.8$ & $4.3 \pm 0.3$ \\
\hline 410245915 & 725.01 & 5.31 & 10.2 & $6173.3 \pm 152.9$ & $0.8 \pm 0.2$ & $4.2 \pm 2.0$ \\
\hline 415969908 & 233.01 & 5.1 & 11.3 & $3644.0 \pm 64.0$ & $0.5 \pm 0.6$ & $4.8 \pm 0.4$ \\
\hline 417676622 & 1290.01 & 2.17 & 9.5 & $5875.2 \pm 137.9$ & $1.3 \pm 3.0$ & $4.3 \pm 0.1$ \\
\hline 417931607 & 1451.01 & 3.53 & 9.0 & $5781.0 \pm 130.5$ & $1.0 \pm 2.1$ & $4.4 \pm 0.1$ \\
\hline 418959198 & 1424.01 & 5.14 & 10.5 & $4529.0 \pm 104.6$ & $0.8 \pm 3.3$ & $4.5 \pm 0.1$ \\
\hline 421894914 & 1056.01 & 4.96 & 9.7 & $6124.3 \pm 83.0$ & $1.4 \pm 1.0$ & $4.4 \pm 0.2$ \\
\hline 424865156 & 1265.01 & 2.82 & 10.0 & $6532.2 \pm 109.2$ & $2.0 \pm 0.7$ & $4.0 \pm 0.1$ \\
\hline 427348923 & 484.01 & 5.54 & 11.6 & $4425.0 \pm 90.0$ & $0.7 \pm 1.2$ & $4.6 \pm 0.1$ \\
\hline 432549364 & 1476.01 & 3.99 & 10.2 & $6596.0 \pm 156.6$ & $1.5 \pm 0.6$ & $4.2 \pm 0.1$ \\
\hline 437242640 & 744.01 & 2.7 & 9.7 & 5700.0 & $0.9 \pm 0.3$ & 4.5 \\
\hline 437248515 & 683.01 & 4.82 & 11.4 & 6302.0 & $1.2 \pm 0.1$ & 4.3 \\
\hline 440100539 & 1548.01 & 2.9 & 8.7 & $5891.9 \pm 92.3$ & $1.2 \pm 0.2$ & $4.3 \pm 0.1$ \\
\hline 445859771 & 1273.01 & 5.9 & 10.4 & $5736.0 \pm 164.8$ & $1.1 \pm 4.6$ & $4.4 \pm 0.1$ \\
\hline 451606970 & 1214.01 & 2.03 & 10.0 & $5262.4 \pm 76.0$ & $1.1 \pm 2.0$ & $4.0 \pm 0.1$ \\
\hline 453211454 & 509.01 & 1.69 & 7.9 & $5560.3 \pm 43.4$ & $1.0 \pm 0.5$ & 4.4 \\
\hline 453260209 & 1215.01 & 6.03 & 9.6 & $3751.0 \pm 68.0$ & $0.5 \pm 1.0$ & $4.8 \pm 0.3$ \\
\hline 455135327 & 490.01 & 4.15 & 9.9 & 6304.0 & $1.2 \pm 0.1$ & 4.4 \\
\hline 458478250 & 1165.01 & 2.79 & 9.8 & $5787.0 \pm 109.9$ & $1.0 \pm 0.6$ & $4.5 \pm 0.1$ \\
\hline 459942762 & 430.01 & 1.86 & 8.2 & $5997.7 \pm 78.3$ & $1.0 \pm 0.1$ & - \\
\hline 459969957 & 1274.01 & 6.28 & 11.9 & $4968.1 \pm 172.4$ & $0.8 \pm 0.5$ & $4.5 \pm 0.1$ \\
\hline 467666275 & 1204.01 & 5.16 & 8.0 & $6711.0 \pm 201.0$ & $1.8 \pm 2.6$ & $4.1 \pm 0.3$ \\
\hline 468148930 & 1086.01 & 5.26 & 12.2 & $5818.0 \pm 190.0$ & $1.1 \pm 8.3$ & $4.3 \pm 2.0$ \\
\hline 1400770435 & 1344.01 & 1.64 & 8.9 & $5941.5 \pm 161.9$ & $1.4 \pm 1.3$ & $4.2 \pm 0.1$ \\
\hline
\end{tabular}

